Interfaces and Free Boundaries 16 (2014), 175-213

DOI 10.4171/IFB/317

\title{
Local solvability and turning for the inhomogeneous Muskat problem
}

\author{
LUIGI C. BERSELLI \\ Dipartimento di matematica, Università di Pisa, via F. Buonarroti 1/c, 56127 Pisa, Italy \\ E-mail: berselli@dma.unipi.it \\ DIEGO CóRDOBA \\ Instituto de Ciencias Matemáticas CSIC-UAM-UC3M-UCM, C/Nicolás Cabrera, 13-15, \\ Campus de Cantoblanco, 28049 Madrid, Spain \\ E-mail:dcg@icmat.es \\ RAFAEL GRANERO-BELINCHÓN \\ Department of Mathematics, University of California, Davis, CA 95616, USA \\ E-mail: rgranero@math.ucdavis.edu
}

[Received 25 January 2013 and in revised form 5 November 2013]

\begin{abstract}
In this work we study the evolution of the free boundary between two different fluids in a porous medium where the permeability is a two dimensional step function. The medium can fill the whole plane $\mathbb{R}^{2}$ or a bounded strip $S=\mathbb{R} \times(-\pi / 2, \pi / 2)$. The system is in the stable regime if the denser fluid is below the lighter one. First, we show local existence in Sobolev spaces by means of energy method when the system is in the stable regime. Then we prove the existence of curves such that they start in the stable regime and in finite time they reach the unstable one. This change of regime (turning) was first proven in [5] for the homogenous Muskat problem with infinite depth.
\end{abstract}

2010 Mathematics Subject Classification: Primary 35R35; Secondary 35Q35.

Keywords: Darcy's law, inhomogeneous Muskat problem, well-posedness, blow-up, maximum principle.

\section{Introduction}

In this work we study the evolution of the interface between two different incompressible fluids with the same viscosity coefficient in a porous medium with two different permeabilities. This problem is of practical importance because it is used as a model for a geothermal reservoir (see [6] and references therein). The velocity of a fluid flowing in a porous medium satisfies Darcy's law $($ see $[3,23,24])$

$$
\frac{\mu}{\kappa(\vec{x})} v=-\nabla p-g \rho(\vec{x})(0,1),
$$

where $\mu$ is the dynamic viscosity, $\kappa(\vec{x})$ is the permeability of the medium, $g$ is the acceleration due to gravity, $\rho(\vec{x})$ is the density of the fluid, $p(\vec{x})$ is the pressure of the fluid and $v(\vec{x})$ is the incompressible velocity field. In our favorite units, we can assume $g=\mu=1$.

The spatial domains considered in this work are $S=\mathbb{R}^{2}, \mathbb{T} \times \mathbb{R}$ (infinite depth) and $\mathbb{R} \times(-\pi / 2, \pi / 2)$ (finite depth). We have two immiscible and incompressible fluids with the same viscosity and different densities; $\rho^{1}$ fill in the upper domain $S^{1}(t)$ and $\rho^{2}$ fill in the lower domain $S^{2}(t)$. The curve

$$
z(\alpha, t)=\left\{\left(z_{1}(\alpha, t), z_{2}(\alpha, t)\right): \alpha \in \mathbb{R}\right\}
$$

(C) European Mathematical Society 2014 
is the interface between the fluids. In particular we are making the ansatz that $S^{1}$ and $S^{2}$ are a partition of $S$ and they are separated by a curve $z$.

The system is in the stable regime if the denser fluid is below the lighter one, i.e. $\rho^{2}>\rho^{1}$. This is known in the literature as the Rayleigh-Taylor condition. The function that measures this condition is defined as

$$
R T(\alpha, t)=-\left(\nabla p^{2}(z(\alpha, t))-\nabla p^{1}(z(\alpha, t))\right) \cdot \partial_{\alpha}^{\perp} z(\alpha, t)>0 .
$$

In the case with $\kappa(\vec{x}) \equiv$ costant $>0$, the motion of a fluid in a two-dimensional porous medium is analogous to the Hele-Shaw cell problem (see [7, 9, 17, 19] and the references therein) and if the fluids fill the whole plane (in the case with the same viscosity but different densities) the contour equation satisfies (see [11])

$$
\partial_{t} f=\frac{\rho^{2}-\rho^{1}}{2 \pi} \text { P.V. } \int_{\mathbb{R}} \frac{\left(\partial_{x} f(x)-\partial_{x} f(x-\eta)\right) \eta}{\eta^{2}+(f(x)-f(x-\eta))^{2}} d \eta .
$$

They show the existence of classical solution locally in time (see [11] and also [1, 15, 16, 20]) in the Rayleigh-Taylor stable regime which means that $\rho^{2}>\rho^{1}$, and maximum principles for $\|f(t)\|_{L^{\infty}}$ and $\left\|\partial_{x} f(t)\right\|_{L^{\infty}}$ (see [12]). Moreover, in [5] the authors show that there exists initial data in $H^{4}$ such that $\left\|\partial_{x} f\right\|_{L^{\infty}}$ blows up in finite time. Furthermore, in [4] the authors prove that there exist analytic initial data in the stable regime for the Muskat problem such that the solution turns to the unstable regime and later no longer belongs to $C^{4}$. In [8] the authors show an energy balance for $L^{2}$ and that if initially $\left\|\partial_{x} f_{0}\right\|_{L^{\infty}}<1$, then there is global Lipschitz solution and if the initial datum has $\left\|f_{0}\right\|_{H^{3}}<1 / 5$ then there is global classical solution. In $[10,27]$ the authors study the case with different viscosities. In [21] the authors study the case where the interface reach the boundary in a moving point with a constant (non-zero) angle.

The case where the fluid domain is the strip $\mathbb{R} \times(-l, l)$, with $0<l$, has been studied in [14-16]. In this regime the equation for the interface is

$$
\begin{aligned}
\partial_{t} f(x, t)=\frac{\rho^{2}-\rho^{1}}{8 l} \text { P.V. } \int_{\mathbb{R}}\left[\frac{\left(\partial_{x} f(x)-\partial_{x} f(x-\eta)\right) \sinh \left(\frac{\pi}{2 l} \eta\right)}{\cosh \left(\frac{\pi}{2 l} \eta\right)-\cos \left(\frac{\pi}{2 l}(f(x)-f(x-\eta))\right)}\right. \\
\left.\quad+\frac{\left(\partial_{x} f(x)+\partial_{x} f(x-\eta)\right) \sinh \left(\frac{\pi}{2 l} \eta\right)}{\cosh \left(\frac{\pi}{2 l} \eta\right)+\cos \left(\frac{\pi}{2 l}(f(x)+f(x-\eta))\right)}\right] d \eta .
\end{aligned}
$$

For equation (1.3) the authors in [14] obtain the existence of classical solution locally in time in the stable regime case where the initial interface does not reach the boundaries, and the existence of finite time singularities. These singularities mean that the curve is initially a graph in the stable regime, and in finite time, the curve can not be parametrized as a graph and the interface turns to the unstable regime. Also the authors study the effect of the boundaries on the evolution of the interface, obtaining the maximum principle and a decay estimate for $\|f\|_{L^{\infty}}$ and the maximum principle for $\left\|\partial_{x} f\right\|_{L^{\infty}}$ for initial datum satisfying smallness conditions on $\left\|\partial_{x} f_{0}\right\|_{L^{\infty}}$ and on $\left\|f_{0}\right\|_{L^{\infty}}$. So, not only the slope must be small, also amplitude of the curve plays a role. Both result differs from the results corresponding to the infinite depth case (1.2). We note that the case with boundaries can also be understood as a problem with different permeabilities where the permeability outside vanishes. 


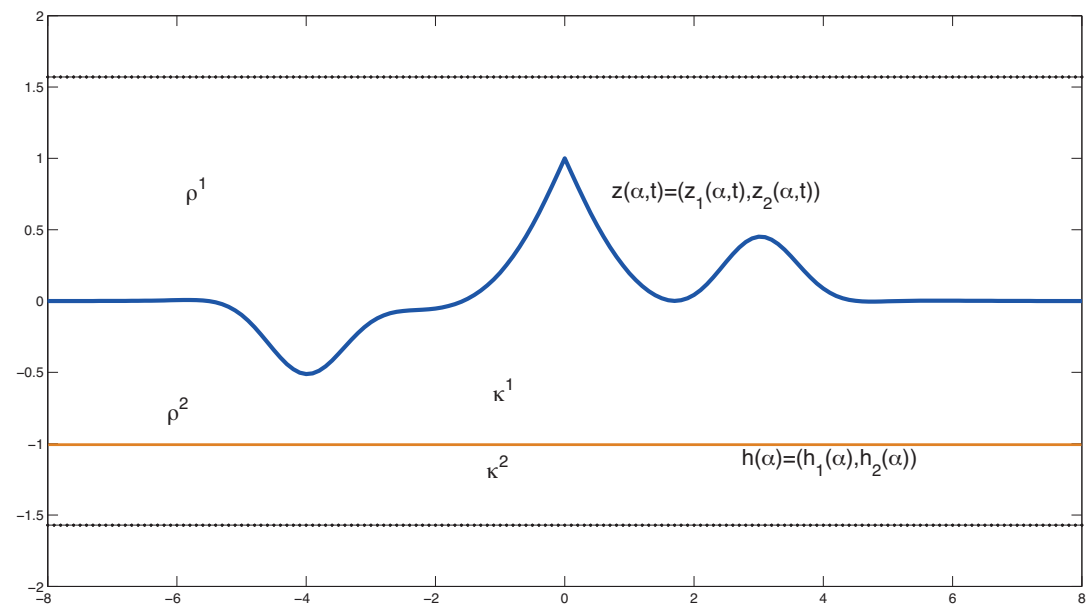

FIG. 1. Physical situation

In the forthcoming work [18] the authors compare the different models (1.2), (1.3) and (1.6) from the point of view of the existence of turning waves.

In this work we study the case where permeability $\kappa(\vec{x})$ is a step function, more precisely, we have a curve

$$
h(\alpha)=\left\{\left(h_{1}(\alpha), h_{2}(\alpha)\right): \alpha \in \mathbb{R}\right\}
$$

separating two regions with different values for the permeability (see Figure 1). We study the regime with infinite depth, for periodic and for "flat at infinity" initial datum, but also the case where the depth is finite and equal to $\frac{\pi}{2}$. In the region above the curve $h(\alpha)$ the permeability is $\kappa(\vec{x}) \equiv \kappa^{1}$, while in the region below the curve $h(\alpha)$ the permeability is $\kappa(\vec{x}) \equiv \kappa^{2} \neq \kappa^{1}$. Note that the curve $h(\alpha)$ is known and fixed. Then it follows from Darcy's law that the vorticity is

$$
\omega(\vec{x})=\varpi_{1}(\alpha, t) \delta(\vec{x}-z(\alpha, t))+\varpi_{2}(\alpha, t) \delta(\vec{x}-h(\alpha)),
$$

where $\varpi_{1}$ corresponds to the difference of the densities, $\varpi_{2}$ corresponding to the difference of permeabilities and $\delta$ is the usual Dirac's distribution. In fact both amplitudes for the vorticity are quite different, while $\varpi_{1}$ is a derivative, the amplitude $\varpi_{2}$ has a nonlocal character (see (1.5), (1.7) and Section 2). The equation for the interface, when $h(x)=\left(x,-h_{2}\right)$ and the fluid fill the whole plane, is

$$
\begin{aligned}
\partial_{t} f(x)=\frac{\kappa^{1}\left(\rho^{2}-\rho^{1}\right)}{2 \pi} \text { P.V. } \int_{\mathbb{R}} & \frac{\left(\partial_{x} f(x)-\partial_{x} f(\beta)\right)(x-\beta)}{(x-\beta)^{2}+(f(x)-f(\beta))^{2}} d \beta \\
& +\frac{1}{2 \pi} \text { P.V. } \int_{\mathbb{R}} \frac{\varpi_{2}(\beta)\left(x-\beta+\partial_{x} f(x)\left(f(x)+h_{2}\right)\right)}{(x-\beta)^{2}+\left(f(x)+h_{2}\right)^{2}} d \beta,
\end{aligned}
$$


with

$$
\varpi_{2}(x)=\frac{\kappa^{1}-\kappa^{2}}{\kappa^{2}+\kappa^{1}} \frac{\kappa^{1}\left(\rho^{2}-\rho^{1}\right)}{\pi} \text { P.V. } \int_{\mathbb{R}} \frac{\partial_{x} f(\beta)\left(h_{2}+f(\beta)\right)}{(x-\beta)^{2}+\left(-h_{2}-f(\beta)\right)^{2}} d \beta
$$

If the fluids fill the whole space but the initial curve is periodic the equation reduces to

$$
\begin{aligned}
\partial_{t} f(x)=\frac{\kappa^{1}\left(\rho^{2}-\rho^{1}\right)}{4 \pi} & \text { P.V. } \int_{\mathbb{T}} \frac{\sin (x-\beta)\left(\partial_{x} f(x)-\partial_{x} f(\beta)\right) d \beta}{\cosh (f(x)-f(\beta))-\cos (x-\beta)} \\
& +\frac{1}{4 \pi} \text { P.V. } \int_{\mathbb{T}} \frac{\left(\partial_{x} f(x) \sinh \left(f(x)+h_{2}\right)+\sin (x-\beta)\right) \varpi_{2}(\beta) d \beta}{\cosh \left(f(x)+h_{2}\right)-\cos (x-\beta)},
\end{aligned}
$$

where the second vorticity amplitude can be written as

$$
\varpi_{2}(x)=\frac{\kappa^{1}\left(\rho^{2}-\rho^{1}\right)}{2 \pi} \frac{\kappa^{1}-\kappa^{2}}{\kappa^{1}+\kappa^{2}} \text { P.V. } \int_{\mathbb{T}} \frac{\sinh \left(h_{2}+f(\beta)\right) \partial_{x} f(\beta) d \beta}{\cosh \left(h_{2}+f(\beta)\right)-\cos (x-\beta)} .
$$

If we consider the regime where the amplitude of the wave and the depth of the medium are of the same order then the equation for the interface, when the depth is chosen to be $\pi / 2$, is

$$
\begin{aligned}
\partial_{t} f(x) & =\frac{\kappa^{1}\left(\rho^{2}-\rho^{1}\right)}{4 \pi} \text { P.V. } \int_{\mathbb{R}} \frac{\left(\partial_{x} f(x)-\partial_{x} f(\beta)\right) \sinh (x-\beta)}{\cosh (x-\beta)-\cos (f(x)-f(\beta))} d \beta \\
& +\frac{\kappa^{1}\left(\rho^{2}-\rho^{1}\right)}{4 \pi} \text { P.V. } \int_{\mathbb{R}} \frac{\left(\partial_{x} f(x)+\partial_{x} f(\beta)\right) \sinh (x-\beta)}{\cosh (x-\beta)+\cos (f(x)+f(\beta))} d \beta \\
& +\frac{1}{4 \pi} \text { P.V. } \int_{\mathbb{R}} \frac{\varpi_{2}(\beta)\left(\sinh (x-\beta)+\partial_{x} f(x) \sin \left(f(x)+h_{2}\right)\right)}{\cosh (x-\beta)-\cos \left(f(x)+h_{2}\right)} d \beta \\
& +\frac{1}{4 \pi} \text { P.V. } \int_{\mathbb{R}} \frac{\varpi_{2}(\beta)\left(-\sinh (x-\beta)+\partial_{x} f(x) \sin \left(f(x)-h_{2}\right)\right)}{\cosh (x-\beta)+\cos \left(f(x)-h_{2}\right)} d \beta,
\end{aligned}
$$

where

$$
\begin{aligned}
& \varpi_{2}(x)=\mathcal{K} \frac{\kappa^{1}\left(\rho^{2}-\rho^{1}\right)}{2 \pi} \text { P.V. } \int_{\mathbb{R}} \partial_{x} f(\beta) \frac{\sin \left(h_{2}+f(\beta)\right)}{\cosh (x-\beta)-\cos \left(h_{2}+f(\beta)\right)} d \beta \\
& -\mathcal{K} \frac{\kappa^{1}\left(\rho^{2}-\rho^{1}\right)}{2 \pi} \text { P.V. } \int_{\mathbb{R}} \partial_{x} f(\beta) \frac{\sin \left(-h_{2}+f(\beta)\right)}{\cosh (x-\beta)+\cos \left(-h_{2}+f(\beta)\right)} d \beta \\
& +\frac{\mathcal{K}^{2}}{\sqrt{2 \pi}} \frac{\kappa^{1}\left(\rho^{2}-\rho^{1}\right)}{2 \pi} G_{h_{2}, \chi} * \text { P.V. } \int_{\mathbb{R}} \frac{\partial_{x} f(\beta) \sin \left(h_{2}+f(\beta)\right)}{\cosh (x-\beta)-\cos \left(h_{2}+f(\beta)\right)} d \beta \\
& -\frac{\mathcal{K}^{2}}{\sqrt{2 \pi}} \frac{\kappa^{1}\left(\rho^{2}-\rho^{1}\right)}{2 \pi} G_{h_{2}, \mathcal{K}} * \text { P.V. } \int_{\mathbb{R}} \frac{\partial_{x} f(\beta) \sin \left(-h_{2}+f(\beta)\right)}{\cosh (x-\beta)+\cos \left(-h_{2}+f(\beta)\right)} d \beta,
\end{aligned}
$$

with

$$
G_{h_{2}, \mathcal{K}}(x)=\mathcal{F}^{-1}\left(\frac{\mathcal{F}\left(\frac{\sin \left(2 h_{2}\right)}{\cosh (x)+\cos \left(2 h_{2}\right)}\right)(\zeta)}{1+\frac{\kappa}{\sqrt{2 \pi}} \mathcal{F}\left(\frac{\sin \left(2 h_{2}\right)}{\cosh (x)+\cos \left(2 h_{2}\right)}\right)(\zeta)}\right)
$$

a Schwartz function. 
REMARK 1 For notational simplicity, we denote $\mathcal{K}=\frac{\kappa^{1}-\kappa^{2}}{\kappa^{1}+\kappa^{2}}$ and we drop the $t$ dependence.

The plan of the paper is as follows: In Section 2 we derive the contour equations (1.4), (1.6) and (1.8). In Section 3 we show the local in time solvability and an energy balance for the $L^{2}$ norm. In Section 4 we perform numerics and in Section 5 we obtain finite time singularities for equations (1.4) (1.6) and (1.8) when the physical parameters are in some region and numerical evidence showing that, in fact, every value is valid for the physical parameters.

\section{The contour equation}

In this section we derive the contour equations (1.4), (1.6) and (1.8), i.e. the equations for the interface. First we obtain the equation in the infinite depth case, both, flat at infinity and periodic. Given $\omega$ a scalar, $\gamma, z$, curves, and a spatial domain $\Omega=\mathbb{T}$ or $\Omega=\mathbb{R}$, we denote the Birkhoff-Rott integral as

$$
B R(\omega, z) \gamma=\text { P.V. } \int_{\Omega} \omega(\beta) B S\left(\gamma_{1}(\alpha), \gamma_{2}(\alpha), z_{1}(\beta), z_{2}(\beta)\right) d \beta,
$$

where $B S$ denotes the kernel of $\nabla^{\perp} \Delta^{-1}$ (which depends on the domain). If the domain is $\mathbb{R}^{2}$ we have

$$
B S(x, y, \mu, v)=\frac{1}{2 \pi}\left(-\frac{y-v}{(y-v)^{2}+(x-\mu)^{2}}, \frac{x-\mu}{(y-v)^{2}+(x-\mu)^{2}}\right),
$$

for $\mathbb{T} \times \mathbb{R}$ we have

$$
B S(x, y, \mu, v)=\frac{1}{4 \pi}\left(\frac{-\sinh (y-v)}{\cosh (y-v)-\cos (x-\mu)}, \frac{\sin (x-\mu)}{\cosh (y-v)-\cos (x-\mu)}\right),
$$

and for $\mathbb{R} \times(-\pi / 2, \pi / 2)$ the kernel is (see [14])

$$
\begin{aligned}
B S(x, y, \mu, v)=\frac{1}{4 \pi}\left(-\frac{\sin (y-v)}{\cosh (x-\mu)-\cos (y-v)}-\frac{\sin (y+v)}{\cosh (x-\mu)+\cos (y+v)},\right. \\
\left.\frac{\sinh (x-\mu)}{\cosh (x-\mu)-\cos (y-v)}-\frac{\sinh (x-\mu)}{\cosh (x-\mu)+\cos (y+v)}\right) .
\end{aligned}
$$

\subsection{Infinite depth}

2.1.1 Assuming $S=\mathbb{R}^{2}: \quad$ Using the kernel (2.2), we obtain

$$
v(\vec{x})=\frac{1}{2 \pi} \text { P.V. } \int_{\mathbb{R}} \varpi_{1}(\beta) \frac{(\vec{x}-z(\beta))^{\perp}}{|\vec{x}-z(\beta)|^{2}} d \beta+\frac{1}{2 \pi} \text { P.V. } \int_{\mathbb{R}} \varpi_{2}(\beta) \frac{(\vec{x}-h(\beta))^{\perp}}{|\vec{x}-h(\beta)|^{2}} d \beta,
$$

where $(a, b)^{\perp}=(-b, a)$.

We have

$$
v^{ \pm}(z(\alpha))=\lim _{\epsilon \rightarrow 0} v\left(z(\alpha) \pm \epsilon \partial_{\alpha}^{\perp} z(\alpha)\right)=B R\left(\varpi_{1}, z\right) z+B R\left(\varpi_{2}, h\right) z \mp \frac{1}{2} \frac{\varpi_{1}(\alpha)}{\left|\partial_{\alpha} z(\alpha)\right|^{2}} \partial_{\alpha} z(\alpha),
$$

and

$$
v^{ \pm}(h(\alpha))=\lim _{\epsilon \rightarrow 0} v\left(h(\alpha) \pm \epsilon \partial_{\alpha}^{\perp} h(\alpha)\right)=B R\left(\varpi_{1}, z\right) h+B R\left(\varpi_{2}, h\right) h \mp \frac{1}{2} \frac{\varpi_{2}(\alpha)}{\left|\partial_{\alpha} h(\alpha)\right|^{2}} \partial_{\alpha} h(\alpha) .
$$


We observe that $v^{+}(z(\alpha))$ is the limit inside $S^{1}$ (the upper subdomain) and $v^{-}(z(\alpha))$ is the limit inside $S^{2}$ (the lower subdomain). The curve $z(\alpha)$ doesn't touch the curve $h(\alpha)$, so, the limit for the curve $h$ are in the same domain $S^{i}$.

Using Darcy's Law and assuming that the initial interface $z(\alpha, 0)$ is in the region with permeability $\kappa^{1}$, we obtain

$$
\begin{aligned}
\left(v^{-}(z(\alpha))-v^{+}(z(\alpha))\right) \cdot \partial_{\alpha} z(\alpha) & =\kappa^{1}\left(-\partial_{\alpha}\left(p^{-}(z(\alpha))-p^{+}(z(\alpha))\right)\right)-\kappa^{1}\left(\rho^{2}-\rho^{1}\right) \partial_{\alpha} z_{1}(\alpha) \\
& =0-\kappa^{1}\left(\rho^{2}-\rho^{1}\right) \partial_{\alpha} z_{2}(\alpha),
\end{aligned}
$$

where in the last equality we have used the continuity of the pressure along the interface (see [10]). Using (2.6) we conclude

$$
\varpi_{1}(\alpha)=-\kappa^{1}\left(\rho^{2}-\rho^{1}\right) \partial_{\alpha} z_{2}(\alpha) .
$$

We need to determine $\varpi_{2}$. We consider

$$
\begin{aligned}
{\left[\frac{v}{\kappa}\right] } & =\left(\frac{v^{-}(h(\alpha))}{\kappa^{2}}-\frac{v^{+}(h(\alpha))}{\kappa^{1}}\right) \cdot \partial_{\alpha} h(\alpha) \\
& =-\partial_{\alpha}\left(p^{-}(h(\alpha))-p^{+}(h(\alpha))\right) \\
& =0,
\end{aligned}
$$

where the first equality is due to Darcy's Law. Using the expression (2.7) we have

$$
\left[\frac{v}{\kappa}\right]=\left(\frac{1}{\kappa^{2}}-\frac{1}{\kappa^{1}}\right)\left(B R\left(\varpi_{1}, z\right) h+B R\left(\varpi_{2}, h\right) h\right) \cdot \partial_{\alpha} h(\alpha)+\left(\frac{1}{2 \kappa^{2}}+\frac{1}{2 \kappa^{1}}\right) \varpi_{2} .
$$

We take $h(\alpha)=\left(\alpha,-h_{2}\right)$, with $h_{2}>0$ a fixed constant. Then

$$
B R\left(\varpi_{2}, h\right) h \cdot \partial_{\alpha} h=\left(0, \frac{1}{2} H\left(\varpi_{2}\right)\right) \cdot(1,0)=0,
$$

where $H$ denotes the Hilbert transform. Finally, we have

$$
\varpi_{2}(\alpha)=-2 \mathcal{K} B R\left(\varpi_{1}, z\right) h \cdot(1,0)=\mathcal{K} \frac{1}{\pi} \mathrm{P} . \mathrm{V} \cdot \int_{\mathbb{R}} \varpi_{1}(\beta) \frac{-h_{2}-z_{2}(\beta)}{|h(\alpha)-z(\beta)|^{2}} d \beta,
$$

(see Remark 1 for the definition of $\mathcal{K}$ ). The identity

$$
\int_{\mathbb{R}} \partial_{\beta} \log \left(\left(A-z_{1}(\beta)\right)^{2}+\left(B-z_{2}(\beta)\right)^{2}\right)=0,
$$

gives us

$$
\frac{1}{2 \pi} \text { P.V. } \int_{\mathbb{R}}\left(-\partial_{\alpha} z_{2}(\beta)\right) \frac{z_{2}(\alpha)-z_{2}(\beta)}{|z(\alpha)-z(\beta)|^{2}} d \beta=\frac{1}{2 \pi} \text { P.V. } \int_{\mathbb{R}} \partial_{\alpha} z_{1}(\beta) \frac{z_{1}(\alpha)-z_{1}(\beta)}{|z(\alpha)-z(\beta)|^{2}} d \beta,
$$

and

$$
\frac{1}{2 \pi} \text { P.V. } \int_{\mathbb{R}} \partial_{\alpha} z_{2}(\beta) \frac{h_{2}+z_{2}(\beta)}{|h(\alpha)-z(\beta)|^{2}} d \beta=\frac{1}{2 \pi} \text { P.V. } \int_{\mathbb{R}} \partial_{\alpha} z_{1}(\beta) \frac{h_{1}(\alpha)-z_{1}(\beta)}{|h(\alpha)-z(\beta)|^{2}} d \beta .
$$


Thus,

$$
\begin{aligned}
\varpi_{2}(\alpha)=\mathcal{K} \frac{\kappa^{1}\left(\rho^{2}-\rho^{1}\right)}{\pi} \text { P.V. } \int_{\mathbb{R}} \partial_{\alpha} z_{2}(\beta) \frac{h_{2}+z_{2}(\beta)}{|h(\alpha)-z(\beta)|^{2}} d \beta \\
=\mathcal{K} \frac{\kappa^{1}\left(\rho^{2}-\rho^{1}\right)}{\pi} \text { P.V. } \int_{\mathbb{R}} \partial_{\alpha} z_{1}(\beta) \frac{h_{1}(\alpha)-z_{1}(\beta)}{|h(\alpha)-z(\beta)|^{2}} d \beta,
\end{aligned}
$$

and

$$
B R\left(\varpi_{1}, z\right) z=\frac{-\kappa^{1}\left(\rho^{2}-\rho^{1}\right)}{2 \pi} \text { P.V. } \int_{\mathbb{R}} \frac{z_{1}(\alpha)-z_{1}(\beta)}{|z(\alpha)-z(\beta)|^{2}} \partial_{\alpha} z(\beta) d \beta
$$

Due to the conservation of mass the curve $z$ is advected by the flow, but we can add any tangential term in the equation for the evolution of the interface without changing the shape of the resulting curve (see [10]), i.e. we consider that the equation for the curve is

$$
\partial_{t} z(\alpha)=v(\alpha)+c(\alpha, t) \partial_{\alpha} z(\alpha) .
$$

Taking $c(\alpha)=-v_{1}(\alpha)$, we conclude

$$
\begin{aligned}
\partial_{t} z=\frac{\kappa^{1}\left(\rho^{2}-\rho^{1}\right)}{2 \pi} \text { P.V. } \int_{\mathbb{R}} & \frac{z_{1}(\alpha)-z_{1}(\beta)}{|z(\alpha)-z(\beta)|^{2}}\left(\partial_{\alpha} z(\alpha)-\partial_{\alpha} z(\beta)\right) d \beta \\
& +\frac{1}{2 \pi} \text { P.V. } \int_{\mathbb{R}} \varpi_{2}(\beta) \frac{(z(\alpha)-h(\beta))^{\perp}}{|z(\alpha)-h(\beta)|^{2}} d \beta \\
& \quad+\partial_{\alpha} z(\alpha) \frac{1}{2 \pi} \text { P.V. } \int_{\mathbb{R}} \varpi_{2}(\beta) \frac{z_{2}(\alpha)+h_{2}}{|z(\alpha)-h(\beta)|^{2}} d \beta .
\end{aligned}
$$

By choosing this tangential term, if our initial datum can be parametrized as a graph, we have $\partial_{t} z_{1}=0$. Therefore the parametrization as a graph propagates.

Finally we conclude (1.4) as the evolution equation for the interface (which initially is a graph above the line $y \equiv-h_{2}$ ). We remark that the second vorticity (1.5) can be written in equivalent ways

$$
\begin{aligned}
\varpi_{2}(x) & =\mathcal{K} \frac{\kappa^{1}\left(\rho^{2}-\rho^{1}\right)}{\pi} \text { P.V. } \int_{\mathbb{R}} \partial_{x} f(\beta) \frac{h_{2}+f(\beta)}{(x-\beta)^{2}+\left(-h_{2}-f(\beta)\right)^{2}} d \beta \\
& =\mathcal{K} \frac{\kappa^{1}\left(\rho^{2}-\rho^{1}\right)}{\pi} \text { P.V. } \int_{\mathbb{R}} \frac{x-\beta}{(x-\beta)^{2}+\left(-h_{2}-f(\beta)\right)^{2}} d \beta \\
& =\mathcal{K} \frac{\kappa^{1}\left(\rho^{2}-\rho^{1}\right)}{2 \pi} \text { P.V. } \int_{\mathbb{R}} \partial_{x} \log \left((x-\beta)^{2}+\left(-h_{2}-f(\beta)\right)^{2}\right) d \beta .
\end{aligned}
$$

REMARK 2 Notice that in the case with different viscosities the expression for the amplitude of the vorticity located at the interface $z(\alpha)$ (see equation (2.8)) is no longer valid. Instead, we have

$$
-\kappa^{1}\left(\rho^{2}-\rho^{1}\right) \partial_{\alpha} z_{2}(\alpha)=\left(\mu^{2}-\mu^{1}\right)\left(B R\left(\varpi_{1}, z\right) z+B R\left(\varpi_{2}, h\right) z\right) \cdot \partial_{\alpha} z(\alpha)+\left(\frac{\mu^{2}+\mu^{1}}{2}\right) \varpi_{1} .
$$

To this integral equation, we add the equation (2.9) or (2.11). Thus, one needs to invert an operator. This is a rather delicate issue that is beyond the scope of this paper (see [10] for further details in the case $\kappa^{1}=\kappa^{2}$ ). 
2.1.2 Assuming $S=\mathbb{T} \times \mathbb{R}$. We have that (2.5) is still valid, but now $\varpi_{i}$ are periodic functions and $z(\alpha+2 k \pi)=z(\alpha)+(2 k \pi, 0)$. Using complex variables notation we have

$$
\begin{aligned}
\bar{v}(\vec{x})=\frac{1}{2 \pi i} \text { P.V. } \int_{\mathbb{R}} \frac{\varpi_{1}(\beta)}{\vec{x}-z(\beta)} d \beta+\frac{1}{2 \pi i} \text { P.V. } \int_{\mathbb{R}} \frac{\varpi_{2}(\beta)}{\vec{x}-h(\beta)} d \beta \\
=\frac{1}{2 \pi i}\left(\text { P.V. } \int_{-\pi}^{\pi}+\sum_{k \geqslant 1}\left(\int_{(2 k-1) \pi}^{(2 k+1) \pi}+\int_{-(2 k+1) \pi}^{-(2 k-1) \pi}\right)\right) \frac{\varpi_{1}(\beta)}{\vec{x}-z(\beta)}+\frac{\varpi_{2}(\beta)}{\vec{x}-h(\beta)} d \beta .
\end{aligned}
$$

Changing variables and using the identity

$$
\frac{1}{z}+\sum_{k \geqslant 1} \frac{2 z}{z^{2}-(2 k \pi)^{2}}=\frac{1}{2 \tan (z / 2)}, \quad \forall z \in \mathbb{C},
$$

we obtain

$$
\bar{v}(\vec{x})=\frac{1}{4 \pi i}\left(\text { P.V. } \int_{\mathbb{T}} \frac{\varpi_{1}(\beta)}{\tan ((\vec{x}-z(\beta)) / 2)} d \beta+\text { P.V. } \int_{\mathbb{T}} \frac{\varpi_{2}(\beta)}{\tan ((\vec{x}-h(\beta)) / 2)} d \beta\right) .
$$

Equivalently,

$$
\begin{aligned}
& v(\vec{x})=\frac{1}{4 \pi}\left(\text { P.V. } \int_{\mathbb{T}}\right. \frac{-\sinh \left(y-z_{2}(\beta)\right) \varpi_{1}(\beta) d \beta}{\cosh \left(y-z_{2}(\beta)\right)-\cos \left(x-z_{1}(\beta)\right)} \\
&\left.+ \text { P.V. } \int_{\mathbb{T}} \frac{-\sinh \left(y-h_{2}(\beta)\right) \varpi_{2}(\beta) d \beta}{\cosh \left(y-h_{2}(\beta)\right)-\cos \left(x-h_{1}(\beta)\right)}\right) \\
&+ \frac{i}{4 \pi}\left(\text { P.V. } \int_{\mathbb{T}} \frac{\sin \left(x-z_{1}(\beta)\right) \varpi_{1}(\beta) d \beta}{\cosh \left(y-z_{2}(\beta)\right)-\cos \left(x-z_{1}(\beta)\right)}\right. \\
&\left.+ \text { P.V. } \int_{\mathbb{T}} \frac{\sin \left(x-h_{1}(\beta)\right) \varpi_{2}(\beta) d \beta}{\cosh \left(y-h_{2}(\beta)\right)-\cos \left(x-h_{1}(\beta)\right)}\right) .
\end{aligned}
$$

Recall that (2.8) and (2.11) are still valid if $h(\alpha)=\left(\alpha,-h_{2}\right)$ for $0<h_{2}$ a fixed constant. We have

$$
\int_{\mathbb{T}} \partial_{\beta} \log \left(\cosh \left(B-z_{2}(\beta)\right)-\cos \left(A-z_{1}(\beta)\right)\right) d \beta=0,
$$

thus, the velocity in the curve when the correct tangential terms are added is

$$
\begin{aligned}
\partial_{t} z(\alpha)= & \frac{1}{4 \pi}\left(\kappa^{1}\left(\rho^{2}-\rho^{1}\right) \text { P.V. } \int_{\mathbb{T}} \frac{\sin \left(z_{1}(\alpha)-z_{1}(\beta)\right)\left(\partial_{\alpha} z(\alpha)-\partial_{\alpha} z(\beta)\right) d \beta}{\cosh \left(z_{2}(\alpha)-z_{2}(\beta)\right)-\cos \left(z_{1}(\alpha)-z_{1}(\beta)\right)}\right. \\
& \left.+\left(\partial_{\alpha} z_{1}(\alpha)-1\right) \text { P.V. } \int_{\mathbb{T}} \frac{\sinh \left(z_{2}(\alpha)+h_{2}\right) \varpi_{2}(\beta) d \beta}{\cosh \left(z_{2}(\alpha)+h_{2}\right)-\cos \left(z_{1}(\alpha)-h_{1}(\beta)\right)}\right) \\
& +\frac{i}{4 \pi} \text { P.V. } \int_{\mathbb{T}} \frac{\left(\partial_{\alpha} z_{2}(\alpha) \sinh \left(z_{2}(\alpha)+h_{2}\right)+\sin \left(z_{1}(\alpha)-h_{1}(\beta)\right)\right) \varpi_{2}(\beta) d \beta}{\cosh \left(z_{2}(\alpha)+h_{2}\right)-\cos \left(z_{1}(\alpha)-h_{1}(\beta)\right)} .
\end{aligned}
$$


We can do the same in order to write $\varpi_{2}$ as an integral on the torus.

$$
\begin{array}{rl}
\varpi_{2}(\alpha)=-2 \mathcal{K} B & R\left(\varpi_{1}, z\right) h \cdot(1,0) \\
= & \frac{1}{2 \pi} \mathcal{K P} . \mathrm{V} \cdot \int_{\mathbb{T}} \frac{\sinh \left(-h_{2}-z_{2}(\beta)\right) \varpi_{1}(\beta) d \beta}{\cosh \left(-h_{2}-z_{2}(\beta)\right)-\cos \left(h_{1}(\alpha)-z_{1}(\beta)\right)} \\
& =\frac{\kappa^{1}\left(\rho^{2}-\rho^{1}\right)}{2 \pi} \mathcal{K P . V} . \int_{\mathbb{T}} \frac{\sinh \left(h_{2}+z_{2}(\beta)\right) \partial_{\alpha} z_{2}(\beta) d \beta}{\cosh \left(-h_{2}-z_{2}(\beta)\right)-\cos \left(h_{1}(\alpha)-z_{1}(\beta)\right)} .
\end{array}
$$

If the initial datum can be parametrized as a graph the equation for the interface reduces to (1.6), where the second vorticity amplitude (1.7) can be written as

$$
\begin{aligned}
\varpi_{2}(x) & =\frac{1}{2 \pi} \mathcal{K P . V} . \int_{\mathbb{T}} \frac{\sinh \left(-h_{2}-f(\beta)\right) \varpi_{1}(\beta) d \beta}{\cosh \left(-h_{2}-f(\beta)\right)-\cos (x-\beta)} \\
& =\frac{\kappa^{1}\left(\rho^{2}-\rho^{1}\right)}{2 \pi} \text { KP.V. } \int_{\mathbb{T}} \frac{\sinh \left(h_{2}+f(\beta)\right) \partial_{x} f(\beta) d \beta}{\cosh \left(h_{2}+f(\beta)\right)-\cos (x-\beta)} \\
& =\frac{\kappa^{1}\left(\rho^{2}-\rho^{1}\right)}{2 \pi} \text { KP.V. } \int_{\mathbb{T}} \frac{\sin (x-\beta) d \beta}{\cosh \left(h_{2}+f(\beta)\right)-\cos (x-\beta)} .
\end{aligned}
$$

\section{$2.2 \quad$ Finite depth}

Now we consider the bounded porous medium $\mathbb{R} \times(-\pi / 2, \pi / 2)$ (see Figure 1$)$. This regime is equivalent to the case with more than two $\kappa^{i}$ because the boundaries can be understood as regions with $\kappa=0$. As before,

$$
v(x, y)=\text { P.V. } \int_{\mathbb{R}} \varpi_{1}(\beta) B S\left(x, y, z_{1}(\beta), z_{2}(\beta)\right) d \beta+\text { P.V. } \int_{\mathbb{R}} \varpi_{2}(\beta) B S\left(x, y, h_{1}(\beta), h_{2}(\beta)\right) d \beta .
$$

We assume that $h(\alpha)=\left(\alpha,-h_{2}\right)$ with $0<h_{2}<\pi / 2$. We have that $\varpi_{1}$ is given by (2.8). The main difference between the finite depth and the infinite depth is at the level of $\varpi_{2}$. As in the infinite depth case we have

$$
0=\left(\frac{1}{\kappa^{2}}-\frac{1}{\kappa^{1}}\right)\left(B R\left(\varpi_{1}, z\right) h+B R\left(\varpi_{2}, h\right) h\right) \cdot \partial_{\alpha} h(\alpha)+\left(\frac{1}{2 \kappa^{2}}+\frac{1}{2 \kappa^{1}}\right) \varpi_{2},
$$

where now $B R$ has the usual definition (2.1) in terms of $B S$ in expression (2.4). In the unbounded case we have an explicit expression for $\varpi_{2}(2.11)$ in terms of $z$ and $h$, but now we have a Fredholm integral equation of second kind:

$$
\varpi_{2}(\alpha)+\frac{\mathcal{K}}{2 \pi} \text { P.V. } \int_{\mathbb{R}} \frac{\varpi_{2}(\beta) \sin \left(2 h_{2}\right)}{\cosh (\alpha-\beta)+\cos \left(2 h_{2}\right)} d \beta=-2 \mathcal{K} B R\left(\varpi_{1}, z\right) h \cdot(1,0) .
$$

After taking the Fourier transform, denoted by $\mathcal{F}(\cdot)(\zeta)$, and using some of its basic properties, we have

$$
\mathcal{F}\left(\varpi_{2}\right)(\zeta)\left(1+\frac{\mathcal{K}}{\sqrt{2 \pi}} \mathcal{F}\left(\frac{\sin \left(2 h_{2}\right)}{\cosh (x)+\cos \left(2 h_{2}\right)}\right)(\zeta)\right)=-2 \mathcal{K} \mathcal{F}\left(B R\left(\varpi_{1}, z\right) h \cdot(1,0)\right)(\zeta) .
$$


We can solve the equation for $\varpi_{2}$ for any $|\mathcal{K}|<\delta\left(h_{2}\right)$ with

$$
\delta\left(h_{2}\right)=\min \left\{1, \frac{\sqrt{2 \pi}}{\max _{\zeta}\left|\mathcal{F}\left(\frac{\sin \left(2 h_{2}\right)}{\cosh (x)+\cos \left(2 h_{2}\right)}\right)\right|}\right\} .
$$

We obtain

$$
\begin{array}{rl}
\varpi_{2}(\alpha)=-2 \mathcal{K} & B R\left(\varpi_{1}, z\right) h \cdot(1,0) \\
+ & \frac{2 \mathcal{K}^{2}}{\sqrt{2 \pi}} B R\left(\varpi_{1}, z\right) h \cdot(1,0) * \mathcal{F}^{-1}\left(\frac{\mathcal{F}\left(\frac{\sin \left(2 h_{2}\right)}{\cosh (x)+\cos \left(2 h_{2}\right)}\right)(\zeta)}{1+\frac{\mathcal{K}}{\sqrt{2 \pi}} \mathcal{F}\left(\frac{\sin \left(2 h_{2}\right)}{\cosh (x)+\cos \left(2 h_{2}\right)}\right)(\zeta)}\right) .
\end{array}
$$

Now we observe that if $s(\zeta)$ is a function in the Schwartz class, $\delta$, such that $1+s(\zeta)>0$ we have that

$$
\frac{s(\zeta)}{1+s(\zeta)} \in \delta
$$

and we obtain

$$
G_{h_{2}, \mathcal{K}}(x)=\mathcal{F}^{-1}\left(\frac{\mathcal{F}\left(\frac{\sin \left(2 h_{2}\right)}{\cosh (x)+\cos \left(2 h_{2}\right)}\right)(\zeta)}{1+\frac{\mathcal{K}}{\sqrt{2 \pi}} \mathcal{F}\left(\frac{\sin \left(2 h_{2}\right)}{\cosh (x)+\cos \left(2 h_{2}\right)}\right)(\zeta)}\right) \in \mathcal{S} .
$$

Recall here that in order to obtain $\varpi_{2}$ we invert an integral operator. In general this is a delicate issue (compare with [10]), but with our choice of $h$ this point can be addressed in a simpler way. Using

$$
\int_{\mathbb{R}} \partial_{\beta} \log \left(\cosh \left(x-z_{1}(\beta)\right) \pm \cos \left(y \pm z_{2}(\beta)\right)\right) d \beta=0,
$$

and adding the correct tangential term, we obtain

$$
\begin{aligned}
\partial_{t} z(\alpha) & =\frac{\kappa^{1}\left(\rho^{2}-\rho^{1}\right)}{4 \pi} \text { P.V. } \int_{\mathbb{R}} \frac{\left(\partial_{\alpha} z(\alpha)-\partial_{\alpha} z(\beta)\right) \sinh \left(z_{1}(\alpha)-z_{1}(\beta)\right)}{\cosh \left(z_{1}(\alpha)-z_{1}(\beta)\right)-\cos \left(z_{2}(\alpha)-z_{2}(\beta)\right)} d \beta \\
& +\frac{\kappa^{1}\left(\rho^{2}-\rho^{1}\right)}{4 \pi} \text { P.V. } \int_{\mathbb{R}} \frac{\left(\partial_{\alpha} z_{1}(\alpha)-\partial_{\alpha} z_{1}(\beta), \partial_{\alpha} z_{2}(\alpha)+\partial_{\alpha} z_{2}(\beta)\right) \sinh \left(z_{1}(\alpha)-z_{1}(\beta)\right)}{\cosh \left(z_{1}(\alpha)-z_{1}(\beta)\right)+\cos \left(z_{2}(\alpha)+z_{2}(\beta)\right)} d \beta \\
& +\frac{1}{4 \pi} \text { P.V. } \int_{\mathbb{R}} \varpi_{2}(\beta) B S\left(z_{1}(\alpha), z_{2}(\alpha), \beta,-h_{2}\right) d \beta \\
& +\frac{\partial_{\alpha} z(\alpha)}{4 \pi} \text { P.V. } \int_{\mathbb{R}} \varpi_{2}(\beta) \frac{\sin \left(z_{2}(\alpha)+h_{2}\right)}{\cosh \left(z_{1}(\alpha)-\beta\right)-\cos \left(z_{2}(\alpha)+h_{2}\right)} d \beta \\
& +\frac{\partial_{\alpha} z(\alpha)}{4 \pi} \text { P.V. } \int_{\mathbb{R}} \varpi_{2}(\beta) \frac{\sin \left(z_{2}(\alpha)-h_{2}\right)}{\cosh \left(z_{1}(\alpha)-\beta\right)+\cos \left(z_{2}(\alpha)-h_{2}\right)} d \beta .
\end{aligned}
$$

If the initial curve can be parametrized as a graph the equation reduces to (1.8) where $\varpi_{2}$ is defined in (1.9).

REMARK 3 If $h_{2}=\pi / 4$ by an explicit computation we obtain $\delta(\pi / 4)=1$, thus, any $\mathcal{K}$ is valid. Moreover, we have tested numerically that the same remains valid for any $0<h_{2}<\pi / 2$, so (1.9) would be correct for any $\mathcal{K}$. 


\section{Well-posedness in Sobolev spaces}

\subsection{Energy balance for the $L^{2}$ norm}

Here we obtain an energy balance inequality for the $L^{2}$ norm of the solution of equation (1.8). We define $\left.\Omega^{1}=\{(x, y), f(x, t)<y<\pi / 2\}, \Omega^{2}=\left\{(x, y),-h_{2}<y<f(x, t)\right)\right\}$ and $\Omega^{3}=$ $\left\{(x, y),-\pi / 2<y<-h_{2}\right\}$.

LEMma 4 For every $0<\kappa^{1}, \kappa^{2}$ the smooth solutions of (1.8) in the stable regime, i.e. $\rho^{2}>\rho^{1}$, case verifies

$$
\|f(t)\|_{L^{2}(\mathbb{R})}^{2}+\int_{0}^{t} \frac{\|v\|_{L^{2}\left(\mathbb{R} \times\left(-h_{2}, \pi / 2\right)\right)}^{2}}{\kappa^{1}\left(\rho^{2}-\rho^{1}\right)}+\frac{\|v\|_{L^{2}\left(\mathbb{R} \times\left(-\pi / 2,-h_{2}\right)\right)}^{2}}{\kappa^{2}\left(\rho^{2}-\rho^{1}\right)} d s=\left\|f_{0}\right\|_{L^{2}(\mathbb{R})}^{2} .
$$

Proof. We define the potentials

$$
\begin{array}{ll}
\phi^{1}(x, y, t)=\kappa^{1}\left(p(x, y, t)+\rho^{1} y\right), & \text { if }(x, y) \in \Omega^{1}, \\
\phi^{2}(x, y, t)=\kappa^{1}\left(p(x, y, t)+\rho^{2} y\right), & \text { if }(x, y) \in \Omega^{2}, \\
\phi^{3}(x, y, t)=\kappa^{2}\left(p(x, y, t)+\rho^{2} y\right), & \text { if }(x, y) \in \Omega^{3} .
\end{array}
$$

We have $v^{i}=-\nabla \phi^{i}$ in each subdomain $S^{i}$. Since the velocity is incompressible we have

$$
0=\int_{\Omega^{i}} \Delta \phi^{i} \phi^{i} d x d y=-\int_{\Omega^{i}}\left|v^{i}\right|^{2} d x d y+\int_{\partial \Omega^{i}} \phi^{i} \partial_{n} \phi^{i} d s .
$$

Moreover, the normal component of the velocity is continuous through the interface $(x, f(x))$ and the line where permeability changes $\left(x,-h_{2}\right)$. Using the impermeable boundary conditions, we only need to integrate over the curve $(x, f(x, t))$ and $\left(x,-h_{2}\right)$. Indeed, we have

$$
\begin{aligned}
0=- & \int_{\Omega^{1}}\left|v^{1}\right|^{2} d x d y \\
& +\kappa^{1} \int_{\mathbb{R}}\left(p(x, f(x, t), t)+\rho^{1} f(x, t)\right)\left(-v(x, f(x, t), t) \cdot\left(\partial_{x} f(x, t),-1\right)\right) d x, \\
0=- & \int_{\Omega^{2}}\left|v^{2}\right|^{2} d x d y \\
& +\kappa^{1} \int_{\mathbb{R}}\left(p(x, f(x, t), t)+\rho^{2} f(x, t)\right)\left(-v(x, f(x, t), t) \cdot\left(-\partial_{x} f(x, t), 1\right)\right) d x \\
& +\kappa^{1} \int_{\mathbb{R}}\left(p\left(x,-h_{2}, t\right)-\rho^{2} h_{2}\right)\left(-v\left(x,-h_{2}, t\right) \cdot(0,-1)\right) d x, \\
0=- & \int_{\Omega^{3}}\left|v^{3}\right|^{2} d x d y \\
& +\kappa^{2} \int_{\mathbb{R}}\left(p\left(x,-h_{2}, t\right)-\rho^{2} h_{2}\right)\left(-v\left(x,-h_{2}, t\right) \cdot(0,1)\right) d x .
\end{aligned}
$$

Inserting (3.4) in (3.3) we get

$$
\begin{aligned}
0= & -\int_{\Omega^{2}}\left|v^{2}\right|^{2} d x d y-\frac{\kappa^{1}}{\kappa^{2}} \int_{\Omega^{3}}\left|v^{3}\right|^{2} d x d y \\
& +\kappa^{1} \int_{\mathbb{R}}\left(p(x, f(x, t), t)+\rho^{2} f(x, t)\right)\left(-v(x, f(x, t), t) \cdot\left(-\partial_{x} f(x, t), 1\right)\right) d x,
\end{aligned}
$$


Thus, summing (3.5) and (3.2) together and using the continuity of the pressure and the velocity in the normal direction, we obtain

$$
\int_{\Omega^{1} \cup \Omega^{2}}|v|^{2} d x d y+\frac{\kappa^{1}}{\kappa^{2}} \int_{\Omega^{3}}|v|^{2} d x d y=\kappa^{1} \int_{\mathbb{R}}\left(\rho^{2}-\rho^{1}\right) f(x, t)\left(-\partial_{t} f(x, t)\right) d x .
$$

Integrating in time we get the desired result (3.1).

\subsection{Well-posedness for the infinite depth case}

Let $\Omega$ be the spatial domain considered, i.e. $\Omega=\mathbb{R}$ or $\Omega=\mathbb{T}$. In this section we prove the short time existence of classical solution for both spatial domains. We have the following result:

TheOREM 3.1 Consider $0<h_{2}$ a fixed constant and the initial datum $f_{0}(x)=f(x, 0) \in H^{k}(\Omega)$, $k \geqslant 3$, such that $-h_{2}<\min _{x} f_{0}(x)$. Then, if the Rayleigh-Taylor condition is satisfied, i.e. $\rho^{2}-$ $\rho^{1}>0$, there exists an unique classical solution of (1.4) $f \in C\left([0, T], H^{k}(\Omega)\right)$ where $T=T\left(f_{0}\right)$. Moreover, we have $f \in C^{1}([0, T], C(\Omega)) \cap C\left([0, T], C^{2}(\Omega)\right)$.

Proof. We prove the result in the case $\Omega=\mathbb{R}$, being the case $\Omega=\mathbb{T}$ similar. Let us consider the usual Sobolev space $H^{3}(\mathbb{R})$ endowed with the norm

$$
\|f\|_{H^{3}}=\|f\|_{L^{2}}+\left\|\Lambda^{3} f\right\|_{L^{2}},
$$

where $\Lambda=\sqrt{-\Delta}$. Define the energy

$$
E[f]:=\|f\|_{H^{3}}+\left\|d^{h}[f]\right\|_{L^{\infty}},
$$

with

$$
d^{h}[f](x, \beta)=\frac{1}{(x-\beta)^{2}+\left(f(x)+h_{2}\right)^{2}} .
$$

To use the classical energy method we need a priori estimates. To simplify notation we drop the physical parameters present in the problem by considering $\kappa^{1}\left(\rho^{2}-\rho^{1}\right)=2 \pi$ and $\mathcal{K}=\frac{1}{2}$. The sign of the difference between the permeabilities will not be important to obtain local existence. We denote $c$ a constant that can changes from one line to another.

Estimates on $\left\|\varpi_{2}\right\|_{H^{3}}$. Given $f(x)$ such that $E[f]<\infty$ we consider $\varpi_{2}$ as defined in (2.13). Then we have that $\left\|\varpi_{2}\right\|_{H^{3}} \leqslant c(E[f]+1)^{k}$ for some constants $c, k$.

We proceed now to prove this claim. We start with the $L^{2}$ norm. Changing variables in (2.13) we have

$$
\begin{aligned}
\left\|\varpi_{2}\right\|_{L^{2}}^{2} \leqslant c \| \text { P.V. } \int_{B(0,1)} \frac{\partial_{x} f(x-\beta)\left(h_{2}+f(x-\beta)\right)}{\beta^{2}+\left(h_{2}+f(x-\beta)\right)^{2}} d \beta \|_{L^{2}}^{2} \\
+c \| \text { P.V. } \int_{B^{c}(0,1)} \frac{\partial_{x} f(x-\beta)\left(h_{2}+f(x-\beta)\right)}{\beta^{2}+\left(h_{2}+f(x-\beta)\right)^{2}} d \beta \|_{L^{2}}^{2} \\
=A_{1}+A_{2} .
\end{aligned}
$$


The inner term, $A_{1}$, can be bounded as follows

$$
\begin{aligned}
A_{1}=\int_{\mathbb{R}} \text { P.V. } \int_{B(0,1)} \frac{\partial_{x} f(x-\beta)\left(h_{2}+f(x-\beta)\right)}{\beta^{2}+\left(h_{2}+f(x-\beta)\right)^{2}} d \beta d x & \\
& \times \text { P.V. } \int_{B(0,1)} \frac{\partial_{x} f(x-\xi)\left(h_{2}+f(x-\xi)\right)}{\xi^{2}+\left(h_{2}+f(x-\xi)\right)^{2}} d \xi d x \\
& \leqslant c\left\|d^{h}[f]\right\|_{L^{\infty}}^{2}\left(1+\|f\|_{L^{\infty}}\right)^{2}\left\|\partial_{x} f\right\|_{L^{2}}^{2} .
\end{aligned}
$$

In the last inequality we have used Cauchy-Schwartz inequality and Tonelli's Theorem. For the outer part we have

$$
\begin{aligned}
& A_{2}=\int_{\mathbb{R}} \text { P.V. } \int_{B^{c}(0,1)} \frac{\partial_{x} f(x-\beta)\left(h_{2}+f(x-\beta)\right)}{\beta^{2}+\left(h_{2}+f(x-\beta)\right)^{2}} d \beta d x \\
& \times \text { P.V. } \int_{B^{c}(0,1)} \frac{\partial_{x} f(x-\xi)\left(h_{2}+f(x-\xi)\right)}{\xi^{2}+\left(h_{2}+f(x-\xi)\right)^{2}} d \xi d x \\
& \leqslant c\left(1+\|f\|_{L^{\infty}}\right)^{2}\left\|\partial_{x} f\right\|_{L^{2}}^{2},
\end{aligned}
$$

where we have used that $\int_{1}^{\infty} \frac{d \beta}{\beta^{2}}<\infty$ and Cauchy-Schwartz inequality. We change variables in (2.14) to obtain

$$
\varpi_{2}(x)=\text { P.V. } \int_{\mathbb{R}} \frac{\beta}{\beta^{2}+\left(h_{2}+f(x-\beta)\right)^{2}} d \beta .
$$

Now it is clear that $\varpi_{2}$ is at the level of $f$ in terms of regularity and the inequality follows using the same techniques. Using Sobolev embedding we conclude this step.

Estimates on $\left\|d^{h}[f]\right\|_{L^{\infty}}$. The first integral in (1.4) can be bounded as follows

$$
I_{1} \leqslant \| \text { P.V. } \int_{\mathbb{R}} \frac{(x-\beta)\left(\partial_{x} f(x)-\partial_{x} f(\beta)\right)}{(x-\beta)^{2}+(f(x)-f(\beta))^{2}} d \beta \|_{L^{\infty}} \leqslant c(E[f]+1)^{k},
$$

for some positive and finite $k$. The new term is the second integral in (1.4).

$$
\begin{aligned}
I_{2} & \leqslant \| \frac{1}{2 \pi} \text { P.V. } \int_{\mathbb{R}} \frac{\varpi_{2}(x-\beta)\left(\beta+\partial_{x} f(x)\left(f(x)+h_{2}\right)\right)}{\beta^{2}+\left(f(x)+h_{2}\right)^{2}} d \beta \|_{L^{\infty}} \\
& \leqslant \| \frac{1}{2 \pi} \text { P.V. } \int_{B(0,1)} d \beta\left\|_{L^{\infty}}+\right\| \frac{1}{2 \pi} \text { P.V. } \int_{B^{c}(0,1)} d \beta \|_{L^{\infty}}=A_{1}+A_{2} .
\end{aligned}
$$

Easily we have

$$
A_{1} \leqslant c\left\|\varpi_{2}\right\|_{L^{\infty}}\left\|d^{h}[f]\right\|_{L^{\infty}}\left(1+\left\|\partial_{x} f\right\|_{L^{\infty}}\left(\|f\|_{L^{\infty}}+1\right)\right) .
$$


We split $A_{2}=B_{1}+B_{2}$

$$
\begin{array}{r}
B_{1}=\frac{1}{2 \pi} \text { P.V. } \int_{B^{c}(0,1)} \frac{\varpi_{2}(x-\beta) \beta}{\beta^{2}+\left(f(x)+h_{2}\right)^{2}} \pm \frac{\varpi_{2}(x-\beta) \beta}{\beta^{2}} d \beta \\
\quad \leqslant c\left\|\varpi_{2}\right\|_{L^{\infty}}\left(\|f\|_{L^{\infty}}+1\right)^{2}+c\left\|H \varpi_{2}\right\|_{L^{\infty}}+c\left\|\partial_{x} \varpi_{2}\right\|_{L^{\infty}},
\end{array}
$$

where $H$ denotes the Hilbert transform. Now we conclude the desired bound using the previous estimate on $\left\|\varpi_{2}\right\|_{H^{3}}$ and Sobolev embedding. The second term can be bounded as

$$
B_{2}=\frac{1}{2 \pi} \text { P.V. } \int_{B^{c}(0,1)} \frac{\varpi_{2}(x-\beta) \partial_{x} f(x)\left(f(x)+h_{2}\right)}{\beta^{2}+\left(f(x)+h_{2}\right)^{2}} d \beta \leqslant c\left\|\varpi_{2}\right\|_{L^{\infty}}\left(\|f\|_{L^{\infty}}+1\right)\left\|\partial_{x} f\right\|_{L^{\infty}}
$$

We obtain the following useful estimate

$$
\left\|\partial_{t} f\right\|_{L^{\infty}} \leqslant c(E[f]+1)^{k}
$$

We have

$$
\frac{d}{d t} d^{h}[f]=\frac{-\partial_{t} f(x) 2\left(f(x)+h_{2}\right)}{\left(\beta^{2}+\left(f(x)+h_{2}\right)^{2}\right)^{2}} \leqslant c d^{h}[f]\left\|d^{h}[f]\right\|_{L^{\infty}}\left(\|f\|_{L^{\infty}}+1\right)\left\|\partial_{t} f\right\|_{L^{\infty}} .
$$

Thus, integrating in time and using (3.9),

$$
\left\|d^{h}[f](t+h)\right\|_{L^{\infty}} \leqslant\left\|d^{h}[f](t)\right\|_{L \infty} e^{c \int_{t}^{t+h}(E[f]+1)^{k}},
$$

and we conclude this step

$$
\frac{d}{d t}\left\|d^{h}[f]\right\|_{L^{\infty}}=\lim _{h \rightarrow 0} \frac{\left\|d^{h}[f](t+h)\right\|_{L^{\infty}}-\left\|d^{h}[f](t)\right\|_{L^{\infty}}}{h} \leqslant c(E[f]+1)^{k}
$$

Estimates on $\left\|\partial_{x}^{3} f\right\|_{L^{2}}$. As before, the bound for the term coming from the first integral in (1.4) can be obtained as in [11], so it only remains the term coming from the second integral. We have

$$
I_{2}=\frac{1}{2 \pi} \int_{\mathbb{R}} \partial_{x}^{3} f(x) \text { P.V. } \int_{\mathbb{R}} \partial_{x}^{3}\left(\frac{\varpi_{2}(x-\beta)\left(\beta+\partial_{x} f(x)\left(f(x)+h_{2}\right)\right)}{\beta^{2}+\left(f(x)+h_{2}\right)^{2}}\right) d \beta d x .
$$

For the sake of brevity we only bound the terms with higher order, being the remaining terms analogous. We have

$$
I_{2}=J_{3}+J_{4}+J_{5}+J_{6}+J_{7}+\text { l.o.t., }
$$


with

$$
\begin{gathered}
J_{3}=\frac{1}{2 \pi} \int_{\mathbb{R}} \partial_{x}^{3} f(x) \text { P.V. } \int_{\mathbb{R}} \frac{\partial_{x}^{3} \varpi_{2}(x-\beta) \beta}{\beta^{2}+\left(f(x)+h_{2}\right)^{2}} d \beta d x, \\
J_{4}=\frac{1}{2 \pi} \int_{\mathbb{R}} \partial_{x}^{3} f(x) \text { P.V. } \int_{\mathbb{R}} \frac{\partial_{x}^{3} \varpi_{2}(x-\beta) \partial_{x} f(x)\left(f(x)+h_{2}\right)}{\beta^{2}+\left(f(x)+h_{2}\right)^{2}} d \beta d x, \\
J_{5}=\frac{1}{2 \pi} \int_{\mathbb{R}} \partial_{x}^{3} f(x) \text { P.V. } \int_{\mathbb{R}} \frac{2 \varpi_{2}(x-\beta)\left(\beta+\partial_{x} f(x)\left(f(x)+h_{2}\right)\right)\left(-f(x)-h_{2}\right) \partial_{x}^{3} f(x)}{\left(\beta^{2}+\left(f(x)+h_{2}\right)^{2}\right)^{2}} d \beta d x, \\
J_{6}=\frac{1}{2 \pi} \int_{\mathbb{R}} \partial_{x}^{3} f(x) \text { P.V. } \int_{\mathbb{R}} \frac{\varpi_{2}(x-\beta)\left(f(x)+h_{2}\right)_{x}^{4} f(x)}{\beta^{2}+\left(f(x)+h_{2}\right)^{2}} d \beta d x,
\end{gathered}
$$

and

$$
J_{7}=\frac{1}{2 \pi} \int_{\mathbb{R}} \partial_{x}^{3} f(x) \text { P.V. } \int_{\mathbb{R}} \frac{4 \varpi_{2}(x-\beta) \partial_{x} f(x) \partial_{x}^{3} f(x)}{\beta^{2}+\left(f(x)+h_{2}\right)^{2}} d \beta d x .
$$

In order to bound $J_{3}$ we use the symmetries in the formulae $\left(\partial_{x}=-\partial_{\beta}\right)$ and we integrate by parts:

$$
\begin{aligned}
J_{3} & =\frac{1}{2 \pi} \int_{\mathbb{R}} \partial_{x}^{3} f(x) \text { P.V. } \int_{\mathbb{R}} \partial_{x}^{2} \varpi_{2}(x-\beta) \partial_{\beta}\left(\frac{\beta}{\beta^{2}+\left(f(x)+h_{2}\right)^{2}}\right) d \beta d x \\
& \leqslant c\left\|\partial_{x}^{3} f\right\|_{L^{2}}\left\|\partial_{x}^{2} \varpi_{2}\right\|_{L^{2}}\left(\left\|d^{h}[f]\right\|_{L^{\infty}}^{2}+\left\|d^{h}[f]\right\|_{L^{\infty}}+1\right) .
\end{aligned}
$$

In $J_{4}$ we use Cauchy-Schwartz inequality to obtain

$$
J_{4} \leqslant c\left(\left\|d^{h}[f]\right\|_{L^{\infty}}+1\right)\left\|\partial_{x}^{3} f\right\|_{L^{2}}\left\|\partial_{x}^{3} \varpi_{2}\right\|_{L^{2}}\left\|\partial_{x} f\right\|_{L^{\infty}}\left(\|f\|_{L^{\infty}}+h_{2}\right)
$$

The bounds for $J_{5}$ and $J_{7}$ are similar:

$$
\begin{gathered}
J_{5} \leqslant c\left(\left\|d^{h}[f]\right\|_{L^{\infty}}^{2}+1\right)\left\|\partial_{x}^{3} f\right\|_{L^{2}}^{2}\left\|\varpi_{2}\right\|_{L^{\infty}}\left(1+\left\|\partial_{x} f\right\|_{L^{\infty}}\left(\|f\|_{L^{\infty}}+h_{2}\right)\right)\left(\|f\|_{L^{\infty}}+h_{2}\right), \\
J_{7} \leqslant c\left(\left\|d^{h}[f]\right\|_{L^{\infty}}+1\right)\left\|\partial_{x}^{3} f\right\|_{L^{2}}^{2}\left\|\varpi_{2}\right\|_{L^{\infty}}\left\|\partial_{x} f\right\|_{L^{\infty}} .
\end{gathered}
$$

Finally, we integrate by parts in $J_{6}$ and we get

$$
\begin{aligned}
J_{6} \leqslant c\left\|\partial_{x}^{3} f\right\|_{L^{2}}^{2}\left(\left\|d^{h}[f]\right\|_{L^{\infty}}+1\right)\left(\left\|\partial_{x} \varpi_{2}\right\|_{L^{\infty}}\left(\|f\|_{L^{\infty}}+1\right)+\left\|\varpi_{2}\right\|_{L^{\infty}}\left\|\partial_{x} f\right\|_{L^{\infty}}\right) \\
+c\left\|\partial_{x}^{3} f\right\|_{L^{2}}^{2}\left(\left\|d^{h}[f]\right\|_{L^{\infty}}^{2}+1\right)\left\|\varpi_{2}\right\|_{L^{\infty}}\left\|\partial_{x} f\right\|_{L^{\infty}}\left(\|f\|_{L^{\infty}}+1\right)^{2} .
\end{aligned}
$$

As a conclusion, we obtain

$$
\frac{d}{d t}\left\|\partial_{x}^{3} f\right\|_{L^{2}} \leqslant c(E[f]+1)^{k} .
$$

Putting all the estimates together we get the desired bound for the energy:

$$
\frac{d}{d t} E[f] \leqslant c(E[f]+1)^{k}
$$


Regularization. This step is classical, so, we only sketch this part (see [22] for the details). We regularize the problem and we show that the regularized problems have a solution using Picard's Theorem on a ball in $H^{3}$. Using the previous energy estimates and the fact that the initial energy is finite, these solutions have the same time of existence ( $T$ depending only on the initial datum) and we can show that they are a Cauchy sequence in $C\left([0, T], L^{2}\right)$. From here we obtain $f \in$ $C\left([0, T], H^{s}(\Omega)\right) \cap L^{\infty}\left([0, T], H^{3}(\Omega)\right)$ where $T=T\left(f_{0}\right)$ and $0<s<3$, a solution to (1.4) as the limit of these regularized solutions. The continuity of the strongest norm $H^{3}$ for positive times follows from the parabolic character of the equation. The continuity of $\|f(t)\|_{H^{3}}$ at $t=0$ follows from the fact that $f(t) \rightarrow f_{0}$ in $H^{3}$ and from the energy estimates.

Uniqueness. Only remains to show that the solution is unique. Let us suppose that for the same initial datum $f_{0}$ there are two smooth solutions $f^{1}$ and $f^{2}$ with finite energy as defined in (3.7) and consider $f=f^{1}-f^{2}$. Following the same ideas as in the energy estimates we obtain

$$
\frac{d}{d t}\|f\|_{L^{2}} \leqslant c\left(f_{0}, E\left[f^{1}\right], E\left[f^{2}\right]\right)\|f\|_{L^{2}} .
$$

Now we conclude using Gronwall inequality.

\subsection{Well-posedness for the finite depth case}

In this section we prove the short time existence of classical solution in the case where the depth is finite. We have the following result:

THEOREM 3.2 Consider $0<h_{2}<\pi / 2$ a constant and $f_{0}(x)=f(x, 0) \in H^{k}(\mathbb{R}), k \geqslant 3$, an initial datum such that $\left\|f_{0}\right\|_{L^{\infty}}<\pi / 2$ and $-h_{2}<\min _{x} f_{0}(x)$. Then, if the Rayleigh-Taylor condition is satisfied, i.e. $\rho^{2}-\rho^{1}>0$, there exists an unique classical solution of $(1.8) f \in C\left([0, T], H^{k}(\mathbb{R})\right)$ where $T=T\left(f_{0}\right)$. Moreover, we have $f \in C^{1}([0, T], C(\mathbb{R})) \cap C\left([0, T], C^{2}(\mathbb{R})\right)$.

Proof. Let us consider the usual Sobolev space $H^{3}(\mathbb{R})$, being the other cases analogous, and define the energy

$$
E[f]=\|f\|_{H^{3}}+\left\|d^{h}[f]\right\|_{L^{\infty}}+\|d[f]\|_{L^{\infty}},
$$

with

$$
d^{h}[f](x, \beta)=\frac{1}{\cosh (x-\beta)-\cos \left(f(x)+h_{2}\right)},
$$

and

$$
d[f](x, \beta)=\frac{1}{\cosh (x-\beta)+\cos (f(x)+f(\beta))} .
$$

We note that $d^{h}[f]$ represents the distance between $f$ and $h$ and $d[f]$ the distance between $f$ and the boundaries. To simplify notation we drop the physical parameters present in the problem by considering $\kappa^{1}\left(\rho^{2}-\rho^{1}\right)=4 \pi$ and $\mathcal{K}=\frac{1}{2}$. Again, the sign of the difference between the permeabilities will not be important to obtain local existence. We write (1.8) as $\partial_{t} f=I_{1}+I_{2}+$ $I_{3}+I_{4}$, being $I_{1}, I_{2}$ the integrals corresponding $\varpi_{1}$ and $I_{3}, I_{4}$ the integrals involving $\varpi_{2}$. We denote $c$ a constant that can changes from one line to another. 
Estimate on $\left\|\varpi_{2}\right\|_{H^{3}}$. Given $f(x)$ such that $E[f]<\infty$ and consider $\varpi_{2}$ as defined in (1.9). Then we have that $\left\|\varpi_{2}\right\|_{H^{3}} \leqslant c(E[f]+1)^{k}$. We need to bound $\left\|J_{1}\right\|_{H^{3}}$ and $\left\|J_{2}\right\|_{H^{3}}$ with

$$
\begin{aligned}
& J_{1}=\text { P.V. } \int_{\mathbb{R}} \partial_{x} f(x-\beta) \frac{\sin \left(h_{2}+f(x-\beta)\right)}{\cosh (\beta)-\cos \left(h_{2}+f(x-\beta)\right)} d \beta \\
& J_{2}=- \text { P.V. } \int_{\mathbb{R}} \partial_{x} f(x-\beta) \frac{\sin \left(-h_{2}+f(x-\beta)\right)}{\cosh (\beta)+\cos \left(-h_{2}+f(x-\beta)\right)} d \beta .
\end{aligned}
$$

We have

$$
\begin{aligned}
\left\|J_{1}\right\|_{L^{2}} \leqslant \| \text { P.V. } \int_{B(0,1)} \frac{\partial_{x} f(x-\beta) \sin \left(h_{2}+f(x-\beta)\right)}{\cosh (\beta)-\cos \left(h_{2}+f(x-\beta)\right)} d \beta \|_{L^{2}} \\
\quad+\| \text { P.V. } \int_{B^{c}(0,1)} \frac{\partial_{x} f(x-\beta) \sin \left(h_{2}+f(x-\beta)\right)}{\cosh (\beta)-\cos \left(h_{2}+f(x-\beta)\right)} d \beta \|_{L^{2}} \\
\leqslant c\left\|\partial_{x} f\right\|_{L^{2}}\left\|d^{h}[f]\right\|_{L^{\infty}}+c\left\|\partial_{x} f\right\|_{L^{2}},
\end{aligned}
$$

where we have used Tonelli's Theorem and Cauchy-Schwartz inequality. Recall that $f-h_{2} \in$ $\left(-2 h_{2}, \frac{\pi}{2}-h_{2}\right)$, thus

$$
\frac{1}{\cosh (x-\beta)+\cos \left(f(x)-h_{2}\right)}<\frac{1}{\cosh (x-\beta)-c\left(h_{2}\right)},
$$

and the kernel corresponding to $\varpi_{2}$ can not be singular and we also obtain

$$
\left\|J_{2}\right\|_{L^{2}} \leqslant c\left\|\partial_{x} f\right\|_{L^{2}} .
$$

Now, as $G_{h_{2}, \varkappa} \in \mathcal{S}$, we can use the Young's inequality for the convolution terms obtaining bounds with an universal constant depending on $h_{2}$ and $\mathcal{K}$. Indeed, we have

$$
\left\|G_{h_{2}, \mathcal{K}} * J_{i}\right\|_{L^{2}} \leqslant c\left\|J_{i}\right\|_{L^{2}}
$$

and we obtain

$$
\left\|\varpi_{2}\right\|_{L^{2}} \leqslant c(E[f]+1)^{k}
$$

Now we observe that

$$
\begin{gathered}
J_{1}=\text { P.V. } \int_{\mathbb{R}} \frac{\sinh (\beta)}{\cosh (\beta)-\cos \left(h_{2}+f(x-\beta)\right)} d \beta, \\
J_{2}=\text { P.V. } \int_{\mathbb{R}} \frac{\sinh (\beta)}{\cosh (\beta)+\cos \left(-h_{2}+f(x-\beta)\right)} d \beta,
\end{gathered}
$$

and we obtain $\left\|\partial_{x}^{3} J_{i}\right\|_{L^{2}} \leqslant c(E[f]+1)^{k}$. Using Young inequality we conclude

$$
\left\|\varpi_{2}\right\|_{H^{3}} \leqslant c(E[f]+1)^{k} .
$$


Estimates on $\left\|d^{h}[f]\right\|_{L^{\infty}}$ and $\|d[f]\|_{L^{\infty}}$. The integrals corresponding to $\varpi_{1}$ in (1.8) can be bounded (see [14]) as

$$
\left|I_{1}+I_{2}\right| \leqslant c(E[f]+1)^{k} .
$$

The new terms are the integrals $I_{3}$ and $I_{4}$, those involving $\varpi_{2}$ in (1.8). We have, when splitted accordingly to the decay at infinity,

$$
I_{3}+I_{4}=J_{3}+J_{4}
$$

where

$$
\begin{aligned}
\left|J_{3}\right| & \leqslant\left\|\frac{1}{4 \pi} \mathrm{P} \cdot \mathrm{V} \cdot \int_{\mathbb{R}} \frac{\varpi_{2}(x-\beta) \sinh (\beta)}{\cosh (\beta)-\cos \left(f(x)+h_{2}\right)}-\frac{\varpi_{2}(x-\beta) \sinh (\beta)}{\cosh (\beta)+\cos \left(f(x)-h_{2}\right)} d \beta\right\|_{L^{\infty}} \\
& \leqslant c\left\|\varpi_{2}\right\|_{L^{\infty}}\left(\left\|d^{h}[f]\right\|_{L^{\infty}}+1\right),
\end{aligned}
$$

and

$$
\begin{aligned}
\left|J_{4}\right| \leqslant \| \frac{1}{4 \pi} \mathrm{P} \cdot \mathrm{V} \cdot \int_{\mathbb{R}} \frac{\varpi_{2}(x-\beta) \partial_{x} f(x) \sin \left(f(x)+h_{2}\right)}{\cosh (\beta)-\cos \left(f(x)+h_{2}\right)} & \\
& \quad+\frac{\varpi_{2}(x-\beta) \partial_{x} f(x) \sin \left(f(x)-h_{2}\right)}{\cosh (\beta)+\cos \left(f(x)-h_{2}\right)} d \beta \|_{L^{\infty}} \\
& \leqslant c\left\|\varpi_{2}\right\|_{L^{\infty}}\left\|\partial_{x} f\right\|_{L^{\infty}}\left(\left\|d^{h}[f]\right\|_{L^{\infty}}+1\right) .
\end{aligned}
$$

We conclude the following useful estimate

$$
\left\|\partial_{t} f\right\|_{L^{\infty}} \leqslant c(E[f]+1)^{k} .
$$

We have

$$
\frac{d}{d t} d^{h}[f]=-\frac{\sin \left(f(x)+h_{2}\right) \partial_{t} f(x)}{\left(\cosh (x-\beta)-\cos \left(f(x)+h_{2}\right)\right)^{2}} \leqslant d^{h}[f]\left\|d^{h}[f]\right\|_{L^{\infty}}\left\|\partial_{t} f\right\|_{L^{\infty}}
$$

Thus, using (3.14) and integrating in time, we obtain the desired bound for $d^{h}[f]$ :

$$
\frac{d}{d t}\left\|d^{h}[f]\right\|_{L^{\infty}}=\lim _{h \rightarrow 0} \frac{\left\|d^{h}[f](t+h)\right\|_{L^{\infty}}-\left\|d^{h}[f](t)\right\|_{L^{\infty}}}{h} \leqslant c(E[f]+1)^{k} .
$$

To obtain the corresponding bound for $d[f]$ we proceed in the same way and we use (3.14) (see [14] for the details).

Estimates on $\left\|\partial_{x}^{3} f\right\|_{L^{2}}$. As before, see [14] for the details concerning the terms coming from $\varpi_{1}$ in (1.8). It only remains the terms coming from $\varpi_{2}$ :

$$
\begin{array}{r}
I=\int_{\mathbb{R}} \text { P.V. } \int_{\mathbb{R}} \partial_{x}^{3} f(x) \partial_{x}^{3}\left(\frac{\varpi_{2}(\beta)\left(\sinh (x-\beta)+\partial_{x} f(x) \sin \left(f(x)+h_{2}\right)\right)}{\cosh (x-\beta)-\cos \left(f(x)+h_{2}\right)}\right. \\
\left.+\frac{\varpi_{2}(\beta)\left(-\sinh (x-\beta)+\partial_{x} f(x) \sin \left(f(x)-h_{2}\right)\right)}{\cosh (x-\beta)+\cos \left(f(x)-h_{2}\right)}\right) d \beta d x .
\end{array}
$$


We split

$$
I=J_{7}+J_{8}+J_{9}+\text { l.o.t.. }
$$

The lower order terms (1.o.t.) can be obtained in a similar way, so we only study the terms $J_{i}$. We have

$$
\begin{aligned}
J_{7} & \leqslant \int_{\mathbb{R}} \text { P.V. } \int_{\mathbb{R}} \frac{\partial_{x}^{3} f(x) \partial_{x}^{3} \varpi_{2}(x-\beta) \sinh (\beta)}{\cosh (\beta)-\cos \left(f(x)+h_{2}\right)}-\frac{\partial_{x}^{3} f(x) \partial_{x}^{3} \varpi_{2}(x-\beta) \sinh (\beta)}{\cosh (\beta)+\cos \left(f(x)-h_{2}\right)} d \beta d x \\
& \leqslant c\left\|\partial_{x}^{3} f\right\|_{L^{2}}\left\|\partial_{x}^{3} \varpi_{2}\right\|_{L^{2}}\left(\left\|d^{h}[f]+1\right\|\right), \\
J_{8} & \leqslant \int_{\mathbb{R}} \text { P.V. } \int_{\mathbb{R}} \frac{\partial_{x}^{3} f(x) \partial_{x}^{3} \varpi_{2}(x-\beta) \partial_{x} f(x) \sin \left(f(x)+h_{2}\right)}{\cosh (\beta)-\cos \left(f(x)+h_{2}\right)} \\
& \leqslant \frac{\partial_{x}^{3} f(x) \partial_{x}^{3} \varpi_{2}(x-\beta) \partial_{x} f(x) \sin \left(f(x)+h_{2}\right)}{\cosh (\beta)+\cos \left(f(x)-h_{2}\right)} d \beta d x \\
& \leqslant c\left\|\partial_{x}^{3} f\right\|_{L^{2}}\left\|\partial_{x}^{3} \varpi_{2}\right\|_{L^{2}}\left\|\partial_{x} f\right\|_{L^{\infty}}\left(\left\|d^{h}[f]+1\right\|\right) .
\end{aligned}
$$

The term $J_{9}$ is given by

$$
\begin{aligned}
J_{9}=\frac{1}{2} \int_{\mathbb{R}} \text { P.V. } \int_{\mathbb{R}} \partial_{x}\left(\partial_{x}^{3} f(x)\right)^{2}\left(\frac{\varpi_{2}(\beta) \sin \left(f(x)+h_{2}\right)}{\cosh (x-\beta)-\cos \left(f(x)+h_{2}\right)}\right. \\
\left.+\frac{\varpi_{2}(\beta) \sin \left(f(x)-h_{2}\right)}{\cosh (x-\beta)+\cos \left(f(x)-h_{2}\right)}\right) d \beta d x .
\end{aligned}
$$

Integrating by parts

$$
\begin{aligned}
\left|J_{9}\right| \leqslant c\left\|\partial_{x}^{3} f\right\|_{L^{2}}\left(\left\|d^{h}[f]\right\|_{L^{\infty}}+1\right) & \left(\left\|\partial_{x} \varpi_{2}\right\|_{L^{\infty}}+\left\|\varpi_{2}\right\|_{L^{\infty}}\left\|\partial_{x} f\right\|_{L^{\infty}}\right) \\
& +c\left\|\partial_{x}^{3} f\right\|_{L^{2}}\left(\left\|d^{h}[f]\right\|_{L^{\infty}}^{2}+1\right)\left\|\varpi_{2}\right\|_{L^{\infty}}\left(1+\left\|\partial_{x} f\right\|_{L^{\infty}}\right)
\end{aligned}
$$

Regularization and uniqueness. These steps follow the same lines as in Theorem 3.1. This concludes the result.

\section{Numerical simulations}

In this section we perform numerical simulations to better understand the role of $\varpi_{2}$. We consider equation (1.6) where $\kappa^{1}=1, \rho^{2}-\rho^{1}=4 \pi$ and $h_{2}=\pi / 2$. For each initial datum we approximate the solution of (1.6) corresponding to different $\mathcal{K}$. Indeed, we take different $\kappa^{2}$ to get $\mathcal{K}=\frac{-999}{1001}, \frac{-1}{3}, 0, \frac{1}{3}$ and $\frac{999}{1001}$.

To perform the simulations we follow the ideas in [13]. The interface is approximated using cubic splines with $N$ spatial nodes. The spatial operator is approximated with Lobatto quadrature (using the function quadl in Matlab). Then, three different integrals appear for a fixed node $x_{i}$. The integral between $x_{i-1}$ and $x_{i}$, the integral between $x_{i}$ and $x_{i+1}$ and the nonsingular ones. In the two first integrals we use Taylor theorem to remove the zeros present in the integrand. In the nonsingular 
integrals the integrand is made explicit using the splines. We use a classical explicit Runge-Kutta method of order 4 to integrate in time. In the simulations we take $N=120$ and $d t=10^{-3}$.

The case 1 (see Figure 2 and 3 ) approximates the solution corresponding to the initial datum

$$
f_{0}(x)=-\left(\frac{\pi}{2}-0.000001\right) e^{-x^{12}} .
$$

The case 2 (see Figures 4 and 5) approximates the solution corresponding to the initial datum

$$
f_{0}(x)=-\left(\frac{\pi}{2}-0.000001\right) \cos \left(x^{2}\right) .
$$

The case 3 (see Figure 6 and 7) approximates the solution corresponding to the initial datum

$$
f_{0}(x)=-\left(\frac{\pi}{2}-0.000001\right) e^{-(x-2)^{12}}-\left(\frac{\pi}{2}-0.000001\right) e^{-(x+2)^{12}}+e^{-x^{2}} \cos ^{2}(x) .
$$

In these simulations we observe that $\|f\|_{C^{1}}$ decays but rather differently depending on $\mathcal{K}$. If $\mathcal{K}<0$ the decay of $\|f\|_{L^{\infty}}$ is faster when compared with the case $\mathcal{K}=0$. In the case where $\mathcal{K}>0$ the term corresponding to $\varpi_{2}$ slows down the decay of $\|f\|_{L^{\infty}}$ but we observe still a decay. Particularly, we observe that if $\mathcal{K} \approx 1\left(\kappa^{2} \approx 0\right)$ the decay is initially almost zero and then slowly increases. When the evolution of $\left\|\partial_{x} f\right\|_{L^{\infty}}$ is considered the situation is reversed. Now the simulations corresponding to $\mathcal{K}>0$ have the faster decay. With these result we can not define a stable regime for $\mathcal{K}$ in which the evolution would be smoother. Recall that we know that there is not any hypothesis on the sign or size of $\mathcal{K}$ to ensure the existence (see Theorem 3.1 and 3.2).

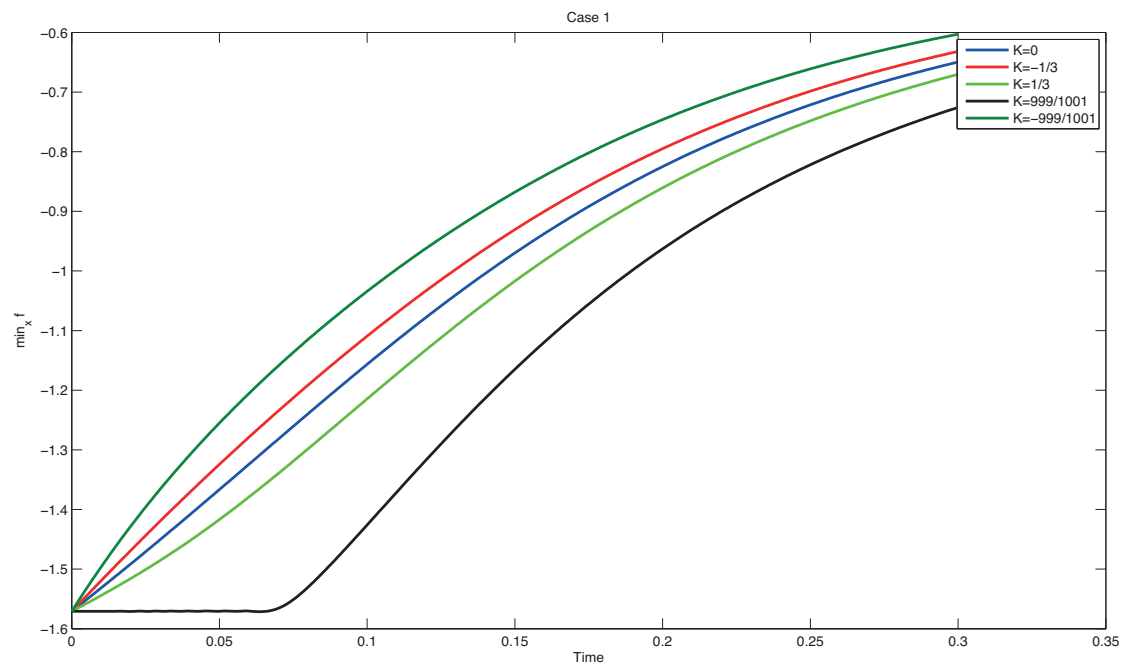

FIG. 2. Evolution of $-\|f\|_{L^{\infty}}$ for different $\mathcal{K}$ in case 1 


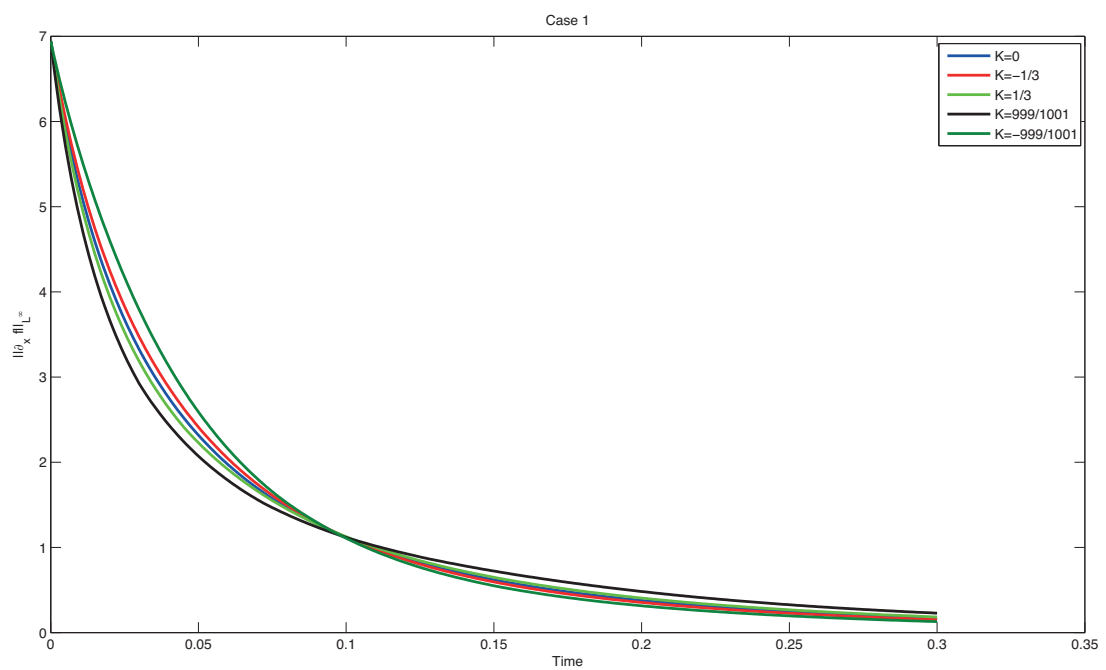

FIG. 3. Evolution of $\left\|\partial_{x} f\right\|_{L^{\infty}}$ for different $\mathcal{K}$ in case 1

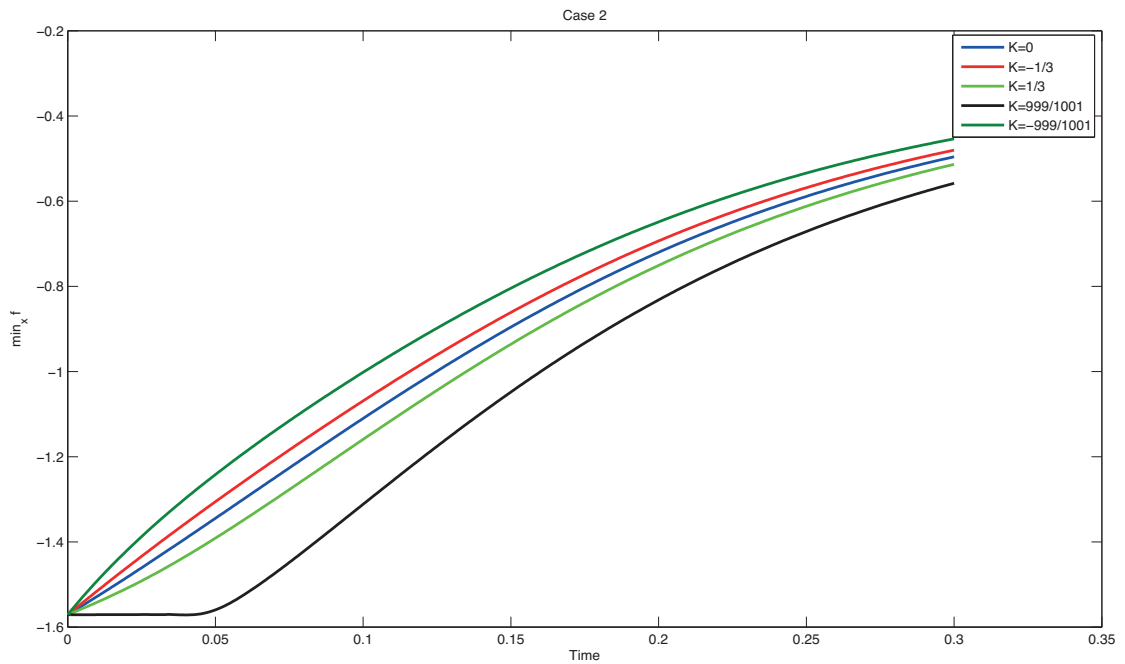

FIG. 4. Evolution of $-\|f\|_{L} \infty$ for different $\mathcal{K}$ in case 2

\section{Turning waves}

In this section we prove finite time singularities for equations (1.4), (1.6) and (1.8). These singularities mean that the curve turns over or, equivalently, in finite time they can not be 


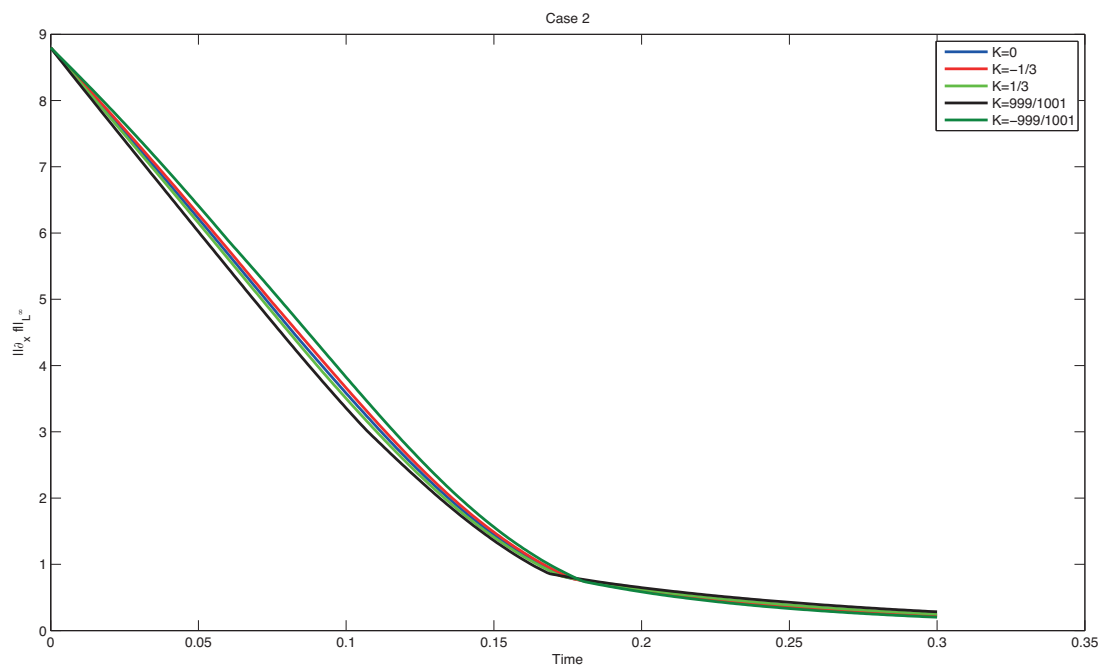

FIG. 5. Evolution of $\left\|\partial_{x} f\right\|_{L^{\infty}}$ for different $\mathcal{K}$ in case 2

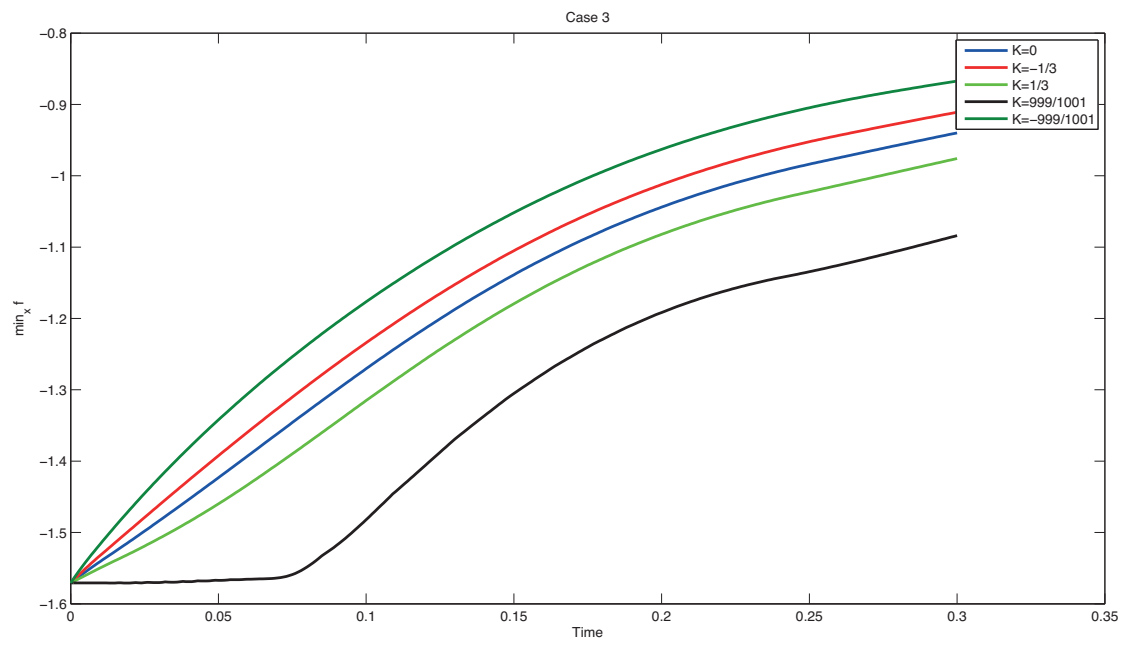

FIG. 6. Evolution of $-\|f\|_{L^{\infty}}$ for different $\mathcal{K}$ in case 3

parametrized as graphs. The proof of turning waves follows the steps and ideas in [5] for the homogenous infinitely deep case where here we have to deal with the difficulties coming from the boundaries and the delta coming from the jump in the permeabilities. 


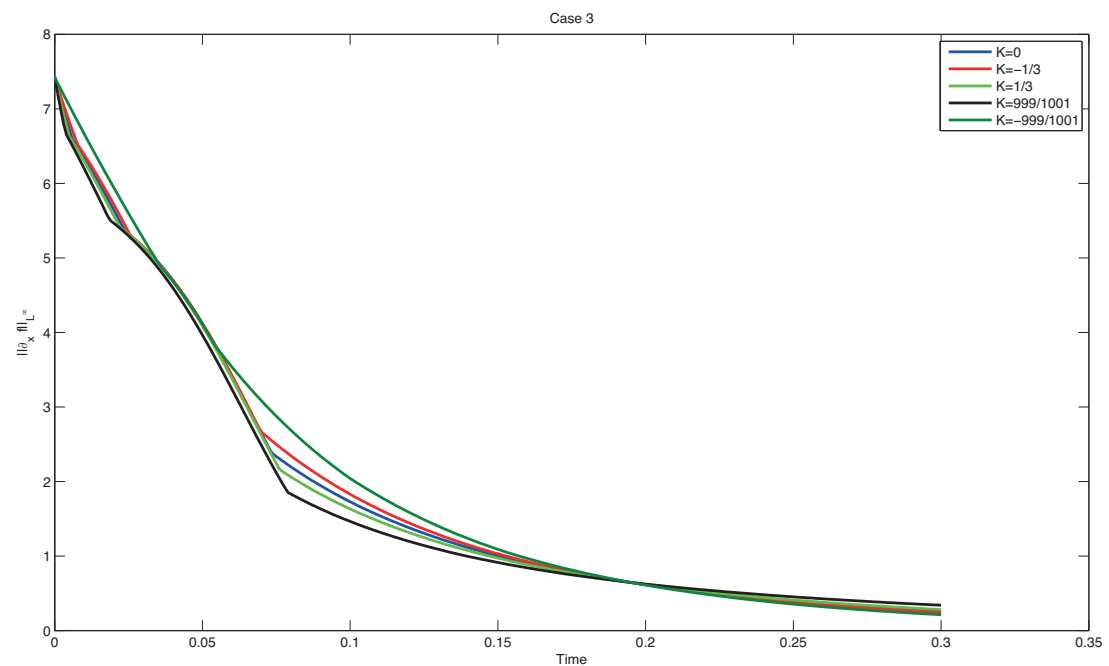

FIG. 7. Evolution of $\left\|\partial_{x} f\right\|_{L^{\infty}}$ for different $\mathcal{K}$ in case 3

\section{$5.1 \quad$ Infinite depth}

Let $\Omega$ be the spatial domain considered, i.e. $\Omega=\mathbb{R}$ or $\Omega=\mathbb{T}$. We have

THEOREM 5.1 Let us suppose that the Rayleigh-Taylor condition is satisfied, i.e. $\rho^{2}-\rho^{1}>0$. Then there exists $f_{0}(x)=f(x, 0) \in H^{3}(\Omega)$, an admissible (see Theorem 3.1) initial datum, such that, for any possible choice of $\kappa^{1}, \kappa^{2}>0$ and $h_{2}>>1$, there exists a solution of (1.4) and (1.6) for which $\lim _{t \rightarrow T^{*}}\left\|\partial_{x} f(t)\right\|_{L^{\infty}}=\infty$ in finite time $0<T^{*}<\infty$. For short time $t>T^{*}$ the solution can be continued but it is not a graph.

Proof. To simplify notation we drop the physical parameters present in the problem by considering $\kappa^{1}\left(\rho^{2}-\rho^{1}\right)=2 \pi$. The proof has three steps. First we consider solutions which are arbitrary curves (not necessary graphs) and we translate the singularity formation to the fact $\partial_{\alpha} v_{1}(0)=$ $\partial_{t} \partial_{\alpha} z_{1}(0)<0$. The second step is to construct a family of curves such that this expression is negative. Thus, we have that if there exists, forward and backward in time, a solution in the RayleighTaylor stable case corresponding to initial data which are arbitrary curves then, we have proved that there is a singularity in finite time. The last step is to prove, using a Cauchy-Kovalevsky theorem, that there exists local in time solutions in this unstable case.

Obtaining the correct expression. Consider the case $\Omega=\mathbb{R}$. Due to (2.12) we have

$$
\partial_{\alpha} \partial_{t} z_{1}(\alpha)=I_{1}+I_{2}+I_{3},
$$


where

$$
\begin{aligned}
& I_{1}(\alpha)=\partial_{\alpha} \text { P.V. } \int_{\mathbb{R}} \frac{z_{1}(\alpha)-z_{1}(\alpha-\beta)}{|z(\alpha)-z(\alpha-\beta)|^{2}}\left(\partial_{\alpha} z_{1}(\alpha)-\partial_{\alpha} z_{1}(\alpha-\beta)\right) d \beta, \\
& I_{2}(\alpha)=\partial_{\alpha} \frac{1}{2 \pi} \text { P.V. } \int_{\mathbb{R}} \varpi_{2}(\alpha-\beta) \frac{-z_{2}(\alpha)-h_{2}}{|z(\alpha)-h(\alpha-\beta)|^{2}} d \beta, \\
& I_{3}(\alpha)=\partial_{\alpha}\left(\partial_{\alpha} z_{1}(\alpha) \frac{1}{2 \pi} \text { P.V. } \int_{\mathbb{R}} \varpi_{2}(\alpha-\beta) \frac{z_{2}(\alpha)+h_{2}}{|z(\alpha)-h(\alpha-\beta)|^{2}} d \beta\right) .
\end{aligned}
$$

Assume now that the following conditions for $z(\alpha)$ holds:

- $z_{i}(\alpha)$ are odd functions,

- $\partial_{\alpha} z_{1}(0)=0, \partial_{\alpha} z_{1}(\alpha)>0 \forall \alpha \neq 0$, and $\partial_{\alpha} z_{2}(0)>0$,

- $z(\alpha) \neq h(\alpha) \forall \alpha$.

The previous hypotheses mean that $z$ is a curve satisfying the arc-chord condition and $\partial_{\alpha} z(0)$ only has vertical component. Due to these conditions on $z$ we have $\partial_{\alpha} z_{1}(0)=0$ and $\partial_{\alpha}^{2} z_{1}$ is odd (and then the second derivative at zero is zero) and we get that $I_{3}(0)=0$. For $I_{1}$ we get

$$
\begin{array}{r}
I_{1}(0)=\text { P.V. } \int_{\mathbb{R}} \frac{\partial_{\alpha}^{2} z_{1}(\beta) z_{1}(\beta)+\left(\partial_{\alpha} z_{1}(\beta)\right)^{2}}{\left(z_{1}(\beta)\right)^{2}+\left(z_{2}(\beta)\right)^{2}} d \beta-2 \mathrm{P} . \mathrm{V} . \int_{\mathbb{R}} \frac{\left(\partial_{\alpha} z_{1}(\beta) z_{1}(\beta)\right)^{2}}{\left(\left(z_{1}(\beta)\right)^{2}+\left(z_{2}(\beta)\right)^{2}\right)^{2}} d \beta \\
+2 \mathrm{P} . \mathrm{V} \cdot \int_{\mathbb{R}} \frac{\partial_{\alpha} z_{1}(\beta) z_{1}(\beta) z_{2}(\beta)\left(\partial_{\alpha} z_{2}(0)-\partial_{\alpha} z_{2}(\beta)\right)}{\left(\left(z_{1}(\beta)\right)^{2}+\left(z_{2}(\beta)\right)^{2}\right)^{2}} d \beta .
\end{array}
$$

We integrate by parts and we obtain, after some lengthy computations,

$$
I_{1}(0)=4 \partial_{\alpha} z_{2}(0) \text { P.V. } \int_{0}^{\infty} \frac{\partial_{\alpha} z_{1}(\beta) z_{1}(\beta) z_{2}(\beta)}{\left(\left(z_{1}(\beta)\right)^{2}+\left(z_{2}(\beta)\right)^{2}\right)^{2}} d \beta
$$

For the term with the second vorticity we have

$$
\begin{aligned}
I_{2}(0)=\frac{1}{2 \pi} \text { P.V. } \int_{\mathbb{R}} \frac{\partial_{\beta} \varpi_{2}(-\beta) h_{2}}{\beta^{2}+h_{2}^{2}} d \beta+\frac{1}{2 \pi} \text { P.V. } \int_{\mathbb{R}} \frac{-\varpi_{2}(-\beta) \partial_{\alpha} z_{2}(0)}{\beta^{2}+h_{2}^{2}} d \beta \\
\quad-\frac{1}{2 \pi} \text { P.V. } \int_{\mathbb{R}} \frac{2 \varpi_{2}(-\beta) \beta h_{2}}{\left(\beta^{2}+h_{2}^{2}\right)^{2}} d \beta-\frac{1}{2 \pi} \text { P.V. } \int_{\mathbb{R}} \frac{\partial_{\alpha} z_{2}(0) \varpi_{2}(-\beta)\left(-h_{2}^{2}\right)}{\left(\beta^{2}+h_{2}^{2}\right)^{2}} d \beta,
\end{aligned}
$$

and, after an integration by parts we obtain

$$
I_{2}(0)=-\frac{\partial_{\alpha} z_{2}(0)}{2 \pi} \text { P.V. } \int_{0}^{\infty} \frac{\left(\varpi_{2}(\beta)+\varpi_{2}(-\beta)\right) \beta^{2}}{\left(\beta^{2}+h_{2}^{2}\right)^{2}} d \beta .
$$


Putting all together we obtain that in the flat at infinity case the important quantity for the singularity is

$$
\begin{array}{r}
\partial_{\alpha} v_{1}(0)=\partial_{\alpha} z_{2}(0)\left(4 \mathrm{P} . \mathrm{V} . \int_{0}^{\infty} \frac{\partial_{\alpha} z_{1}(\beta) z_{1}(\beta) z_{2}(\beta)}{\left(\left(z_{1}(\beta)\right)^{2}+\left(z_{2}(\beta)\right)^{2}\right)^{2}} d \beta\right. \\
\left.\quad-\frac{1}{2 \pi} \text { P.V. } \int_{0}^{\infty} \frac{\left(\varpi_{2}(\beta)+\varpi_{2}(-\beta)\right) \beta^{2}}{\left(\beta^{2}+h_{2}^{2}\right)^{2}} d \beta\right),
\end{array}
$$

where, due to (2.11), $\varpi_{2}$ is defined as

$$
\varpi_{2}(\beta)=2 \text { KP.V. } \int_{\mathbb{R}} \frac{\left(h_{2}+z_{2}(\gamma)\right) \partial_{\alpha} z_{2}(\gamma)}{\left(h_{2}+z_{2}(\gamma)\right)^{2}+\left(\beta-z_{1}(\gamma)\right)^{2}} d \gamma .
$$

We apply the same procedure to equation (2.15) and we get the important quantity in the periodic setting (recall the superscript $p$ in the notation denoting that we are in the periodic setting):

$$
\begin{aligned}
\partial_{\alpha} v_{1}^{p}(0)=\partial_{\alpha} z_{2}(0)\left(\int_{0}^{\pi} \frac{\partial_{\alpha} z_{1}(\beta) \sin \left(z_{1}(\beta)\right) \sinh \left(z_{2}(\beta)\right)}{\left(\cosh \left(z_{2}(\beta)\right)-\cos \left(z_{1}(\beta)\right)\right)^{2}} d \beta\right. \\
\left.\quad+\frac{1}{4 \pi} \int_{0}^{\pi} \frac{\left(\varpi_{2}^{p}(\beta)+\varpi_{2}^{p}(-\beta)\right)\left(-1+\cosh \left(h_{2}\right) \cos (\beta)\right)}{\left(\cosh \left(h_{2}\right)-\cos (\beta)\right)^{2}} d \beta\right),
\end{aligned}
$$

and, due to (2.16),

$$
\varpi_{2}^{p}(\beta)=\mathcal{K} \int_{\mathbb{T}} \frac{\sin \left(\beta-z_{1}(\gamma)\right) \partial_{\alpha} z_{1}(\gamma)}{\cosh \left(h_{2}+z_{2}(\gamma)\right)-\cos \left(\beta-z_{1}(\gamma)\right)} d \gamma .
$$

Taking the appropriate curve. To clarify the proof, let us consider first the periodic setting. Given $1<h_{2}$, we consider $a, b$, constants such that $2<b \leqslant a$ and let us define

$$
z_{1}(\alpha)=\alpha-\sin (\alpha)
$$

and

$$
z_{2}(\alpha)= \begin{cases}\frac{\sin (a \alpha)}{a} & \text { if } 0 \leqslant \alpha \leqslant \frac{\pi}{a}, \\ \frac{\sin \left(\pi \frac{\alpha-(\pi / a)}{(\pi / a)-(\pi / b)}\right)}{b} & \text { if } \frac{\pi}{a}<\alpha<\frac{\pi}{b}, \\ \left(\frac{-h_{2} / 2}{\left.\frac{\pi}{2}-\frac{\pi}{b}\right)\left(\alpha-\frac{\pi}{b}\right)}\right. & \text { if } \frac{\pi}{b} \leqslant \alpha<\frac{\pi}{2}, \\ -\left(\frac{-h_{2} / 2}{\left.\frac{\pi}{2}-\frac{\pi}{b}\right)\left(\alpha-\pi+\frac{\pi}{b}\right)}\right. & \text { if } \frac{\pi}{2} \leqslant \alpha<\pi\left(1-\frac{1}{b}\right), \\ 0 & \text { if } \pi\left(1-\frac{1}{b}\right) \leqslant \alpha .\end{cases}
$$




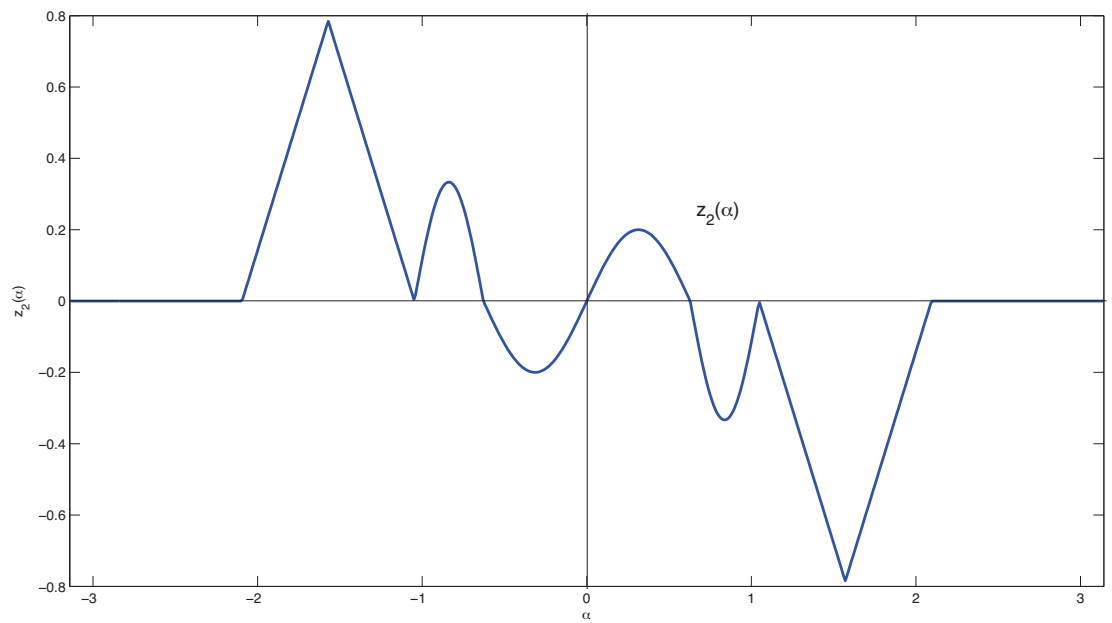

FIG. 8. $z_{2}$ in (5.7) for $a=5, b=3, h_{2}=\pi / 2$

Due to the definition of $z_{2}$, we have

$$
\frac{h_{2}}{2} \leqslant h_{2}+z_{2}(\alpha) \leqslant \frac{3 h_{2}}{2},
$$

and using (5.6), we get

$$
\left|\varpi_{2}^{p}(\beta)\right| \leqslant \frac{4 \pi}{\cosh \left(h_{2} / 2\right)-1} .
$$

Inserting this curve in (5.5) we obtain

$$
\partial_{\alpha} v_{1}^{p}(0) \leqslant I_{a}+I_{b}^{h_{2}}+I_{2}^{h_{2}},
$$

with

$$
\begin{aligned}
I_{a}=\int_{0}^{\pi / a} \frac{(1-\cos (\beta)) \sin (\beta-\sin (\beta)) \sinh \left(\frac{\sin (a \beta)}{a}\right)}{\left(\cosh \left(\frac{\sin (a \beta)}{a}\right)-\cos (\beta-\sin (\beta))\right)^{2}} d \beta, \\
I_{b}^{h_{2}}=\int_{(\pi / b+\pi) / 3}^{\pi / 2} \frac{(1-\cos (\beta)) \sin (\beta-\sin (\beta)) \sinh \left(\left(\frac{-h_{2} / 2}{\frac{\pi}{2}-\frac{\pi}{b}}\right)\left(\beta-\frac{\pi}{b}\right)\right)}{\left(\cosh \left(\left(\frac{-h_{2} / 2}{\frac{\pi}{2}-\frac{\pi}{b}}\right)\left(\beta-\frac{\pi}{b}\right)\right)-\cos (\beta-\sin (\beta))\right)^{2}} d \beta \\
+\int_{\pi / 2}^{(2 \pi-\pi / b) / 3)} \frac{(1-\cos (\beta)) \sin (\beta-\sin (\beta)) \sinh \left(-\left(\frac{-h_{2} / 2}{\frac{\pi}{2}-\frac{\pi}{b}}\right)\left(\alpha-\pi+\frac{\pi}{b}\right)\right)}{\left(\cosh \left(-\left(\frac{-h_{2} / 2}{\frac{\pi}{2}-\frac{\pi}{b}}\left(\alpha-\pi+\frac{\pi}{b}\right)\right)\right)-\cos (\beta-\sin (\beta))\right)^{2}} d \beta,
\end{aligned}
$$


and $I_{2}^{h_{2}}$ is the integral involving the second vorticity $\varpi_{2}^{p}$. We remark that $I_{a}$ does not depend on $h_{2}$. The sign of $I_{b}^{h_{2}}$ is the same as the sign of $z_{2}$, thus we get $I_{b}^{h_{2}}<0$ and this is independent of the choice of $a$ and $h_{2}$. Now we fix $b$ and we take $h_{2}$ sufficiently large such that

$$
I_{b}^{h_{2}}+I_{2}^{h_{2}} \leqslant I_{b}^{h_{2}}+\frac{2 \pi}{\cosh \left(h_{2} / 2\right)-1} \frac{1+\cosh \left(h_{2}\right)}{\left(\cosh \left(h_{2}\right)-1\right)^{2}}<0 .
$$

We can do that because

$$
\frac{c_{b} \sinh \left(h_{2} / 3\right)}{\left(\cosh \left(h_{2} / 2\right)+1\right)^{2}} \leqslant\left|I_{b}^{h_{2}}\right|
$$

or, equivalently,

$$
I_{b}^{h_{2}}+I_{2}^{h_{2}}=-\left|I_{b}^{h_{2}}\right|+I_{2}^{h_{2}} \leqslant-\frac{c_{b} \sinh \left(h_{2} / 3\right)}{\left(\cosh \left(h_{2} / 2\right)-1\right)^{2}}+\frac{2 \pi}{\cosh \left(h_{2} / 2\right)-1} \frac{1+\cosh \left(h_{2}\right)}{\left(\cosh \left(h_{2}\right)-1\right)^{2}}<0,
$$

if $h_{2}$ is large enough. The integral $I_{a}$ is well defined and positive, but goes to zero as $a$ grows. Then, fixed $b$ and $h_{2}$ in such a way $I_{b}^{h_{2}}+I_{2}^{h_{2}}<0$, we take $a$ sufficiently large such that $I_{a}+I_{b}^{h_{2}}+I_{2}^{h_{2}}<0$. We are done with the periodic case.

We proceed with the flat at infinity case. We take $2<b \leqslant a$ as before and $0<\delta<1$ and define

$$
z_{1}(\alpha)=\alpha-\sin (\alpha) \exp \left(-\alpha^{2}\right),
$$

and

$$
z_{2}(\alpha)= \begin{cases}\frac{\sin (a \alpha)}{a} & \text { if } 0 \leqslant \alpha \leqslant \frac{\pi}{a}, \\ \frac{\sin \left(\pi \frac{\alpha-(\pi / a)}{(\pi / a)-(\pi / b)}\right)}{b} & \text { if } \frac{\pi}{a}<\alpha<\frac{\pi}{b}, \\ \left(\frac{-h_{2}^{\delta}}{\left.\frac{\pi}{2}-\frac{\pi}{b}\right)\left(\alpha-\frac{\pi}{b}\right)}\right. & \text { if } \frac{\pi}{b} \leqslant \alpha<\frac{\pi}{2}, \\ -\left(\frac{-h_{2}^{\delta}}{\frac{\pi}{2}-\frac{\pi}{b}}\right)\left(\alpha-\pi+\frac{\pi}{b}\right) & \text { if } \frac{\pi}{2} \leqslant \alpha<\pi\left(1-\frac{1}{b}\right), \\ 0 & \text { if } \pi\left(1-\frac{1}{b}\right) \leqslant \alpha .\end{cases}
$$

We have

$$
h_{2}-h_{2}^{\delta}<h_{2}+z_{2}(\beta)<h_{2}+h_{2}^{\delta},
$$

and we assume $1<h_{2}-h_{2}^{\delta}$. Inserting the curve (5.8) and (5.9) in (5.4) and changing variables, we obtain

$$
\left|\varpi_{2}(\beta)\right| \leqslant 2 \text { P.V. } \int_{\mathbb{R}} \frac{\left(h_{2}+h_{2}^{\delta}\right) h_{2}^{\delta}\left(\frac{\pi}{2}-\frac{\pi}{b}\right)^{-1}}{\left(h_{2}-h_{2}^{\delta}\right)^{2}+\left(\gamma-\sin (\beta-\gamma) e^{-(\beta-\gamma)^{2}}\right)^{2}} d \gamma .
$$

We split the integral in two parts:

$$
J_{1}=2 \text { P.V. } \int_{B\left(0,2\left(h_{2}-h_{2}^{\delta}\right)\right)} \frac{\left(h_{2}+h_{2}^{\delta}\right) h_{2}^{\delta}\left(\frac{\pi}{2}-\frac{\pi}{b}\right)^{-1}}{\left(h_{2}-h_{2}^{\delta}\right)^{2}+\left(\gamma-\sin (\beta-\gamma) e^{-(\beta-\gamma)^{2}}\right)^{2}} d \gamma \leqslant 8 h_{2}^{\delta}\left(\frac{\pi}{2}-\frac{\pi}{b}\right)^{-1},
$$


and

$$
J_{2}=2 \mathrm{P} . \mathrm{V} . \int_{B^{c}\left(0,2\left(h_{2}-h_{2}^{\delta}\right)\right)} \frac{\left(h_{2}+h_{2}^{\delta}\right) h_{2}^{\delta}\left(\frac{\pi}{2}-\frac{\pi}{b}\right)^{-1}}{\left(h_{2}-h_{2}^{\delta}\right)^{2}+\left(\gamma-\sin (\beta-\gamma) e^{-(\beta-\gamma)^{2}}\right)^{2}} d \gamma
$$

We have

$$
\begin{aligned}
K_{1} & =\text { P.V. } \int_{2\left(h_{2}-h_{2}^{\delta}\right)}^{\infty} \frac{1}{\left(h_{2}-h_{2}^{\delta}\right)^{2}+\left(\gamma-\sin (\beta-\gamma) e^{-(\beta-\gamma)^{2}}\right)^{2}} d \gamma \\
& \leqslant \text { P.V. } \int_{2\left(h_{2}-h_{2}^{\delta}\right)}^{\infty} \frac{1}{\left(h_{2}-h_{2}^{\delta}\right)^{2}+\gamma^{2}-2 \gamma \sin (\beta-\gamma) e^{-(\beta-\gamma)^{2}}} d \gamma \\
& \leqslant \text { P.V. } \int_{2\left(h_{2}-h_{2}^{\delta}\right)}^{\infty} \frac{1}{\left(h_{2}-h_{2}^{\delta}-\gamma\right)^{2}+2 \gamma\left(h_{2}-h_{2}^{\delta}-\sin (\beta-\gamma) e^{-(\beta-\gamma)^{2}}\right)} d \gamma,
\end{aligned}
$$

and using that $h_{2}$ is such that $1<h_{2}-h_{2}^{\delta}$, we get

$$
K_{1} \leqslant \text { P.V. } \int_{2\left(h_{2}-h_{2}^{\delta}\right)}^{\infty} \frac{1}{\left(h_{2}-h_{2}^{\delta}-\gamma\right)^{2}} d \gamma=\frac{1}{h_{2}-h_{2}^{\delta}} .
$$

The remaining integral is

$$
\begin{aligned}
K_{2}=\text { P.V. } \int_{-\infty}^{-2\left(h_{2}-h_{2}^{\delta}\right)} \frac{1}{\left(h_{2}-h_{2}^{\delta}\right)^{2}+\left(\gamma-\sin (\beta-\gamma) e^{-(\beta-\gamma)^{2}}\right)^{2}} d \gamma \\
\quad \leqslant \text { P.V. } \int_{-\infty}^{-2\left(h_{2}-h_{2}^{\delta}\right)} \frac{1}{\left(h_{2}-h_{2}^{\delta}\right)^{2}+\gamma^{2}-2 \gamma \sin (\beta-\gamma) e^{-(\beta-\gamma)^{2}}} d \gamma \\
\quad \leqslant \text { P.V. } \int_{-\infty}^{-2\left(h_{2}-h_{2}^{\delta}\right)} \frac{1}{\left(h_{2}-h_{2}^{\delta}+\gamma\right)^{2}-2 \gamma\left(h_{2}-h_{2}^{\delta}+\sin (\beta-\gamma) e^{-(\beta-\gamma)^{2}}\right)} d \gamma,
\end{aligned}
$$

and using that $h_{2}$ is such that $1<h_{2}-h_{2}^{\delta}$, we get

$$
K_{2} \leqslant \text { P.V. } \int_{-\infty}^{-2\left(h_{2}-h_{2}^{\delta}\right)} \frac{1}{\left(h_{2}-h_{2}^{\delta}+\gamma\right)^{2}} d \gamma=\frac{1}{h_{2}-h_{2}^{\delta}}
$$

Putting all together we get

$$
J_{2} \leqslant 4 h_{2}^{\delta}\left(\frac{\pi}{2}-\frac{\pi}{b}\right)^{-1},
$$

and

$$
\left|\varpi_{2}(\beta)\right| \leqslant 12 h_{2}^{\delta}\left(\frac{\pi}{2}-\frac{\pi}{b}\right)^{-1} .
$$

Using this bound in (5.3) we get

$$
\left|I_{2}^{h_{2}}\right| \leqslant 3 h_{2}^{\delta-1}\left(\frac{\pi}{2}-\frac{\pi}{b}\right)^{-1} .
$$

Then, as before,

$$
\partial_{\alpha} v_{1}(0) \leqslant I_{a}+I_{b}^{h_{2}}+I_{2}^{h_{2}},
$$


where $I_{a}, I_{b}^{h_{2}}$ are the integrals $I_{1}(0)$ on the intervals $(0, \pi / a)$ and $((\pi / b+\pi) / 3,(2 \pi-\pi / b) / 3)$, respectively. We have

$$
c_{b} \frac{2 h_{2}^{\delta}}{3\left(h_{2}^{\delta}\right)^{4}} \leqslant\left|I_{b}^{h_{2}}\right|
$$

thus,

$$
I_{b}^{h_{2}}+I_{2}^{h_{2}}=-\left|I_{b}^{h_{2}}\right|+I_{2}^{h_{2}} \leqslant-c_{b} \frac{2 h_{2}^{-3 \delta}}{3}+3 h_{2}^{\delta-1}\left(\frac{\pi}{2}-\frac{\pi}{b}\right)^{-1} .
$$

To ensure that the decay of $I_{2}^{h_{2}}$ is faster than the decay of $I_{b}^{h_{2}}$ we take $\delta<1 / 4$. Now, fixing $b$, we can obtain $1<h_{2}$ and $0<\delta<1 / 4$ such that $1<h_{2}-h_{2}^{\delta}$ and $I_{b}^{h_{2}}+I_{2}^{h_{2}}<0$. Taking $a>>b$ we obtain a curve such that $\partial_{\alpha} v_{1}(0)<0$. In order to conclude the argument it is enough to approximate these curves (5.7) and (5.9) by analytic functions. We are done with this step of the proof.

Showing the forward and backward solvability. At this point, we need to prove that there is a solution forward and backward in time corresponding to these curves (5.7) and (5.9). Indeed, if this solution exists then, due to the previous step, we obtain that, for a short time $t<0$, the solution is a graph with finite $H^{3}(\Omega)$ energy (in fact, it is analytic). This graph at time $t=0$ has a blow up for $\left\|\partial_{x} f\right\|_{L^{\infty}}$ and, for a short time $t>0$, the solution can not be parametrized as a graph. We show the result corresponding to the flat at infinity case, being the periodic one analogous. We consider curves $z$ satisfying the arc-chord condition and such that

$$
\lim _{|\alpha| \rightarrow \infty}|z(\alpha)-(\alpha, 0)|=0
$$

We define the complex strip $\mathbb{B}_{r}=\{\zeta+i \xi, \zeta \in \mathbb{R},|\xi|<r\}$, and the spaces

$$
X_{r}=\left\{z=\left(z_{1}, z_{2}\right) \text { analytic curves satisfying the arc-chord condition on } \mathbb{B}_{r}\right\} \text {, }
$$

with norm

$$
\|z\|_{r}^{2}=\|z(\gamma)-(\gamma, 0)\|_{H^{3}\left(\mathbb{B}_{r}\right)}^{2},
$$

where $H^{3}\left(\mathbb{B}_{r}\right)$ denotes the Hardy-Sobolev space on the strip with the norm

$$
\|f\|_{r}^{2}=\sum_{ \pm} \int_{\mathbb{R}}|f(\zeta \pm r i)|^{2} d \zeta+\int_{\mathbb{R}}\left|\partial_{\alpha}^{3} f(\zeta \pm r i)\right|^{2} d \zeta
$$

(see [2]). These spaces form a Banach scale. For notational convenience we write $\gamma=\alpha \pm i r$, $\gamma^{\prime}=\alpha \pm i r^{\prime}$. Recall that, for $0<r^{\prime}<r$,

$$
\left\|\partial_{\alpha} \cdot\right\|_{L^{2}\left(\mathbb{B}_{r^{\prime}}\right)} \leqslant \frac{C}{r-r^{\prime}}\|\cdot\|_{L^{2}\left(\mathbb{B}_{r}\right)} .
$$


We consider the complex extension of (2.11) and (2.12), which is given by

$$
\begin{gathered}
\partial_{t} z(\gamma)=\text { P.V. } \int_{\mathbb{R}} \frac{\left(z_{1}(\gamma)-z_{1}(\gamma-\beta)\right)\left(\partial_{\alpha} z(\gamma)-\partial_{\alpha} z(\gamma-\beta)\right)}{\left(z_{1}(\gamma)-z_{1}(\gamma-\beta)\right)^{2}+\left(z_{2}(\gamma)-z_{2}(\gamma-\beta)\right)^{2}} d \beta \\
\quad+\frac{1}{2 \pi} \text { P.V. } \int_{\mathbb{R}} \frac{\varpi_{2}(\gamma-\beta)(z(\gamma)-h(\gamma-\beta))^{\perp}}{\left(z_{1}(\gamma)-(\gamma-\beta)\right)^{2}+\left(z_{2}(\gamma)+h_{2}\right)^{2}} d \beta \\
\quad+\partial_{\alpha} z(\gamma) \frac{1}{2 \pi} \text { P.V. } \int_{\mathbb{R}} \frac{\left(z_{2}(\gamma)+h_{2}\right) \varpi_{2}(\gamma-\beta)}{\left(z_{1}(\gamma)-(\gamma-\beta)\right)^{2}+\left(z_{2}(\gamma)+h_{2}\right)^{2}} d \beta,
\end{gathered}
$$

with

$$
\varpi_{2}(\gamma)=2 \mathcal{K} \text { P.V. } \int_{\mathbb{R}} \frac{\left(h_{2}+z_{2}(\gamma-\beta)\right) \partial_{\alpha} z_{2}(\gamma-\beta)}{\left(\gamma-z_{1}(\gamma-\beta)\right)^{2}+\left(h_{2}+z_{2}(\gamma-\beta)\right)^{2}} d \beta .
$$

Recall the fact that in the case of a real variable graph $\varpi_{2}$ has the same regularity as $f$, but in the case of an arbitrary curve $\varpi_{2}$ is, roughly speaking, at the level of the first derivative of the interface. This fact will be used below. We define

$$
\begin{gathered}
d^{-}[z](\gamma, \beta)=\frac{\beta^{2}}{\left(z_{1}(\gamma)-z_{1}(\gamma-\beta)\right)^{2}+\left(z_{2}(\gamma)-z_{2}(\gamma-\beta)\right)^{2}}, \\
d^{h}[z](\gamma, \beta)=\frac{1+\beta^{2}}{\left(z_{1}(\gamma)-(\gamma-\beta)\right)^{2}+\left(z_{2}(\gamma)+h_{2}\right)^{2}} .
\end{gathered}
$$

The function $d^{-}$is the complex extension of the arc chord condition and we need it to bound the terms with $\varpi_{1}$. The function $d^{h}$ comes from the different permeabilities and we use it to bound the terms with $\varpi_{2}$. We observe that both are bounded functions for the considered curves. Consider $0<r^{\prime}<r$ and the set

$$
O_{R}=\left\{z \in X_{r} \text { such that }\|z\|_{r}<R,\left\|d^{-}[z]\right\|_{L^{\infty}\left(\mathbb{B}_{r}\right)}<R,\left\|d^{h}[z]\right\|_{L^{\infty}\left(\mathbb{B}_{r}\right)}<R\right\},
$$

where $d^{-}[z]$ and $d^{h}[z]$ are defined in (5.15) and (5.16). Then we claim that, for $z, w \in O_{R}$, the right-hand side of (5.13), $F: O_{R} \rightarrow X_{r^{\prime}}$ is continuous and the following inequalities holds:

$$
\begin{gathered}
\|F[z]\|_{H^{3}\left(\mathbb{B}_{r^{\prime}}\right)} \leqslant \frac{C_{R}}{r-r^{\prime}}\|z\|_{r}, \\
\|F[z]-F[w]\|_{H^{3}\left(\mathbb{B}_{r^{\prime}}\right)} \leqslant \frac{C_{R}}{r-r^{\prime}}\|z-w\|_{H^{3}\left(\mathbb{B}_{r}\right)}, \\
\sup _{\gamma \in \mathbb{B}_{r}, \beta \in \mathbb{R}}|F[z](\gamma)-F[z](\gamma-\beta)| \leqslant C_{R}|\beta| .
\end{gathered}
$$

The claim for the spatial operator corresponding to $\varpi_{1}$ has been studied in [5], thus, we only deal with the new terms containing $\varpi_{2}$. For the sake of brevity we only bound some terms, being the other analogous. Using Tonelli's theorem and Cauchy-Schwartz inequality we have that

$$
\left\|\varpi_{2}\right\|_{L^{2}\left(\mathbb{B}_{r^{\prime}}\right)} \leqslant c\left\|d^{h}[z]\right\|_{L^{\infty}}\left(1+\left\|z_{2}\right\|_{L^{\infty}\left(\mathbb{B}_{r^{\prime}}\right)}\right)\left\|\partial_{\alpha} z_{2}\right\|_{L^{2}\left(\mathbb{B}_{r^{\prime}}\right)} .
$$


Moreover, we get

$$
\left\|\varpi_{2}\right\|_{H^{2}\left(\mathbb{B}_{r}\right)} \leqslant C_{R}\|z\|_{r} .
$$

For $\partial_{\alpha}^{3} \varpi_{2}$ the procedure is similar but we lose one derivative. Using (5.12) and Sobolev embedding we conclude

$$
\left\|\varpi_{2}\right\|_{H^{3}\left(\mathbb{B}_{r^{\prime}}\right)} \leqslant \frac{C_{R}}{r-r^{\prime}}\|z\|_{r} .
$$

From here inequality (5.17) follows. Inequality (5.18), for the terms involving $\varpi_{1}$, can be obtained using the properties of the Hilbert transform as in [5]. Let's change slightly the notation and write $\varpi_{2}[z](\gamma)$ for the integral in (5.14). We split

$$
\begin{aligned}
A_{1}=\text { P.V. } \int_{\mathbb{R}} \frac{\left(\varpi_{2}[z]\left(\gamma^{\prime}-\beta\right)-\varpi_{2}[w]\left(\gamma^{\prime}-\beta\right)\right)\left(z\left(\gamma^{\prime}\right)-h\left(\gamma^{\prime}-\beta\right)\right)^{\perp}}{\left(z_{1}\left(\gamma^{\prime}\right)-\left(\gamma^{\prime}-\beta\right)\right)^{2}+\left(z_{2}\left(\gamma^{\prime}\right)+h_{2}\right)^{2}} d \beta \\
+ \text { P.V. } \int_{\mathbb{R}} \frac{\varpi_{2}[w]\left(\gamma^{\prime}-\beta\right)\left(\left(z\left(\gamma^{\prime}\right)-h\left(\gamma^{\prime}-\beta\right)\right)^{\perp}-\left(w\left(\gamma^{\prime}\right)-h\left(\gamma^{\prime}-\beta\right)\right)^{\perp}\right)}{\left(z_{1}\left(\gamma^{\prime}\right)-\left(\gamma^{\prime}-\beta\right)\right)^{2}+\left(z_{2}\left(\gamma^{\prime}\right)+h_{2}\right)^{2}} d \beta \\
\quad+\text { P.V. } \int_{\mathbb{R}} \varpi_{2}[w]\left(\gamma^{\prime}-\beta\right)\left(w\left(\gamma^{\prime}\right)-h\left(\gamma^{\prime}-\beta\right)\right)^{\perp} \frac{d^{h}[z]\left(\gamma^{\prime}, \beta\right)-d^{h}[w]\left(\gamma^{\prime}, \beta\right)}{1+\beta^{2}} d \beta \\
=B_{1}+B_{2}+B_{3} .
\end{aligned}
$$

In $B_{3}$ we need some extra decay at infinity to ensure the finiteness of the integral. We compute

$$
\left|d^{h}[z]-d^{h}[w]\right| \leqslant C_{R} \frac{\left|d^{h}[z] d^{h}[w]\right|}{1+\beta^{2}}\left|(1+\beta)\left(z_{1}-w_{1}\right)+z_{2}-w_{2}\right|<C_{R}|z-w| \frac{|1+\beta|}{1+\beta^{2}},
$$

and, due to Sobolev embedding, we get

$$
\left\|B_{3}\right\|_{L^{2}\left(\mathbb{B}_{r^{\prime}}\right)} \leqslant C_{R}\left\|\varpi_{2}[w]\right\|_{L^{2}\left(\mathbb{B}_{r^{\prime}}\right)}\|z-w\|_{L^{\infty}\left(\mathbb{B}_{r^{\prime}}\right)} \leqslant \frac{C_{R}}{r-r^{\prime}}\|z-w\|_{H^{3}\left(\mathbb{B}_{r}\right)} .
$$

For the second term we obtain the same bound

$$
\left\|B_{2}\right\|_{L^{2}\left(\mathbb{B}_{r^{\prime}}\right)} \leqslant C_{R}\left\|\varpi_{2}[w]\right\|_{L^{2}\left(\mathbb{B}_{r^{\prime}}\right)}\|z-w\|_{L^{\infty}\left(\mathbb{B}_{r^{\prime}}\right)} \leqslant \frac{C_{R}}{r-r^{\prime}}\|z-w\|_{H^{3}\left(\mathbb{B}_{r}\right)} .
$$

We split $B_{1}$ componentwise. In the first coordinate we have

$$
\begin{aligned}
\left\|C_{1}\right\|_{L^{2}\left(\mathbb{B}_{r^{\prime}}\right)} & =\| \text { P.V. } \int_{\mathbb{R}} \frac{\left(\varpi_{2}[z]\left(\gamma^{\prime}-\beta\right)-\varpi_{2}[w]\left(\gamma^{\prime}-\beta\right)\right)\left(-z_{2}\left(\gamma^{\prime}\right)-h_{2}\right)}{\left(z_{1}\left(\gamma^{\prime}\right)-\left(\gamma^{\prime}-\beta\right)\right)^{2}+\left(z_{2}\left(\gamma^{\prime}\right)+h_{2}\right)^{2}} d \beta \|_{L^{2}\left(\mathbb{B}_{r^{\prime}}\right)} \\
& \leqslant C_{R}\left\|\varpi_{2}[z]-\varpi_{2}[w]\right\|_{L^{2}\left(\mathbb{B}_{r^{\prime}}\right)} .
\end{aligned}
$$

In the second coordinate we need to ensure the integrability at infinity. We get

$$
\begin{aligned}
C_{2}=\text { P.V. } & \int_{\mathbb{R}} \frac{\left(\varpi_{2}[z]\left(\gamma^{\prime}-\beta\right)-\varpi_{2}[w]\left(\gamma^{\prime}-\beta\right)\right)\left(z_{1}\left(\gamma^{\prime}\right)-\gamma^{\prime}\right)}{\left(z_{1}\left(\gamma^{\prime}\right)-\left(\gamma^{\prime}-\beta\right)\right)^{2}+\left(z_{2}\left(\gamma^{\prime}\right)+h_{2}\right)^{2}} d \beta \\
+ \text { P.V. } & \int_{\mathbb{R}}\left(\varpi_{2}[z]\left(\gamma^{\prime}-\beta\right)-\varpi_{2}[w]\left(\gamma^{\prime}-\beta\right)\right)\left(\frac{\beta d^{h}[z]}{1+\beta^{2}}-\frac{1}{\beta}\right) d \beta \\
& +H \varpi_{2}[z]\left(\gamma^{\prime}\right)-H \varpi_{2}[w]\left(\gamma^{\prime}\right),
\end{aligned}
$$


and, with this splitting and the properties of the Hilbert transform, we obtain

$$
\left\|C_{2}\right\|_{L^{2}\left(\mathbb{B}_{r^{\prime}}\right)} \leqslant C_{R}\left\|\varpi_{2}[z]-\varpi_{2}[w]\right\|_{L^{2}\left(\mathbb{B}_{r^{\prime}}\right)} .
$$

We get

$$
\varpi_{2}[z]-\varpi_{2}[w]=C_{3}+C_{4}+C_{5},
$$

where

$$
\begin{aligned}
& C_{3}=2 \mathcal{K} \text { P.V. } \int_{\mathbb{R}} \frac{\left(z_{2}(\gamma-\beta)-w_{2}(\gamma-\beta)\right) \partial_{\alpha} z_{2}(\gamma-\beta)}{\left(\gamma-z_{1}(\gamma-\beta)\right)^{2}+\left(h_{2}+z_{2}(\gamma-\beta)\right)^{2}} d \beta, \\
& C_{4}=2 \mathcal{K} \text { P.V. } \int_{\mathbb{R}} \frac{\left(h_{2}+w_{2}(\gamma-\beta)\right)\left(\partial_{\alpha} z_{2}(\gamma-\beta)-\partial_{\alpha} w_{2}(\gamma-\beta)\right)}{\left(\gamma-z_{1}(\gamma-\beta)\right)^{2}+\left(h_{2}+z_{2}(\gamma-\beta)\right)^{2}} d \beta,
\end{aligned}
$$

and

$$
C_{5}=2 \mathcal{K} \text { P.V. } \int_{\mathbb{R}}\left(h_{2}+w_{2}(\gamma-\beta)\right) \partial_{\alpha} w_{2}(\gamma-\beta) \frac{d^{h}[z](\gamma-\beta,-\beta)-d^{h}[w](\gamma-\beta,-\beta)}{1+\beta^{2}} d \beta .
$$

From these expressions we obtain

$$
\begin{aligned}
&\left\|C_{3}\right\|_{L^{2}\left(\mathbb{B}_{r^{\prime}}\right)} \leqslant C_{R}\|z-w\|_{L^{\infty}}\left\|\partial_{\alpha} z_{2}\right\|_{L^{2}\left(\mathbb{B}_{r^{\prime}}\right)}, \\
&\left\|C_{4}\right\|_{L^{2}\left(\mathbb{B}_{r^{\prime}}\right)} \leqslant C_{R}\left\|\partial_{\alpha}(z-w)\right\|_{L^{2}\left(\mathbb{B}_{r^{\prime}}\right)},
\end{aligned}
$$

and

$$
\left\|C_{5}\right\|_{L^{2}\left(\mathbb{B}_{r^{\prime}}\right)} \leqslant C_{R}\|z-w\|_{L^{\infty}}\left\|\partial_{\alpha} z_{2}\right\|_{L^{2}\left(\mathbb{B}_{r^{\prime}}\right)}
$$

Collecting all these estimates, and due to Sobolev embedding and (5.12) we obtain

$$
\left\|B_{1}\right\|_{L^{2}\left(\mathbb{B}_{r^{\prime}}\right)} \frac{C_{R}}{r-r^{\prime}}\|z-w\|_{H^{3}\left(\mathbb{B}_{r}\right)} .
$$

We are done with (5.18). Inequality (5.19) is equivalent to the bound $\left|\partial_{t} \partial_{\alpha} z\right|<C_{R}$. Such a bound for the terms involving $\varpi_{2}$ can be obtained from (5.16) and (5.20). For instance

$$
\begin{aligned}
A_{2}=\text { P.V. } \int_{\mathbb{R}} \frac{\partial_{\alpha} \varpi_{2}(\gamma-\beta)(z(\gamma)-h(\gamma-\beta))^{\perp}}{\left(z_{1}(\gamma)-(\gamma-\beta)\right)^{2}+\left(z_{2}(\gamma)+h_{2}\right)^{2}} d \beta= \\
\quad-\mathrm{P} . \mathrm{V} \cdot \int_{\mathbb{R}} \varpi_{2}(\gamma-\beta) \partial_{\beta}\left(\frac{(z(\gamma)-h(\gamma-\beta))^{\perp}}{\left(z_{1}(\gamma)-(\gamma-\beta)\right)^{2}+\left(z_{2}(\gamma)+h_{2}\right)^{2}}\right) d \beta \\
\quad \leqslant C_{R}\left\|\varpi_{2}\right\|_{H^{2}\left(\mathbb{B}_{r}\right)}\left\|d^{h}[z]\right\|_{L^{\infty}} .
\end{aligned}
$$

The remaining terms can be handled in a similar way. Now we can finish with the forward and backward solvability step. Take $z(0)$ the analytic extension of $z$ in (5.9) ((5.7) for the periodic case). We have $z(0) \in X_{r_{0}}$ for some $r_{0}>0$, it satisfies the arc-chord condition and does not reach 
the curve $h$, thus, there exists $R_{0}$ such that $z(0) \in O_{R_{0}}$. We take $r<r_{0}$ and $R>R_{0}$ in order to define $O_{R}$ and we consider the iterates

$$
z_{n+1}=z(0)+\int_{0}^{t} F\left[z_{n}\right] d s, z_{0}=z(0),
$$

and assume by induction that $z_{k} \in O_{R}$ for $k \leqslant n$. Then, following the proofs in [5, 14, 25, 26], we obtain a time $T_{C K}>0$ of existence. It remains to show that

$$
\left\|d^{-}\left[z_{n+1}\right]\right\|_{L^{\infty}\left(\mathbb{B}_{r}\right)}, \quad\left\|d^{h}\left[z_{n+1}\right]\right\|_{L^{\infty}\left(\mathbb{B}_{r}\right)}<R,
$$

for some times $T_{A}, T_{B}>0$ respectively. Then we choose $T=\min \left\{T_{C K}, T_{A}, T_{B}\right\}$ and we finish the proof. As $d^{-}$has been studied in [5] we only deal with $d^{h}$. Due to (5.17) and the definition of $z(0)$, we have

$$
\left(d^{+}\left[z_{n+1}\right]\right)^{-1}>\frac{1}{R_{0}}-C_{R}\left(t^{2}+t\right),
$$

and, if we take a sufficiently small $T_{B}$ we can ensure that for $t<T_{B}$ we have $d^{h}\left[z_{n+1}\right]<R$. We conclude the proof of the Theorem.

We observe that in the periodic case the curve $z$ is of the same order as $h_{2}$, so, even if $h_{2}>>1$, this result is not some kind of linearization. The same result is valid if $\mathcal{K}<<1$ for any $h_{2}$ (see Theorem 5.2). Moreover, we have numerical evidence showing that for every $|\mathcal{K}|<1$ and $h_{2}=\pi / 2$ (and not $h_{2}>>1$ ) there are curves showing turning effect.

Numerical Evidence 5 There are curves such that for every $|\mathcal{K}|<1$ and $h_{2}=\pi / 2$ turn over.

Let us consider first the periodic setting. Recall the fact that $h_{2}=\pi / 2$ and let us define

$$
z_{1}(\alpha)=\alpha-\sin (\alpha), \quad z_{2}(\alpha)=\frac{\sin (3 \alpha)}{3}-\sin (\alpha)\left(e^{-(\alpha+2)^{2}}+e^{-(\alpha-2)^{2}}\right) \text { for } \alpha \in \mathbb{T} .
$$

Inserting this curve in (5.5) we obtain that for any possible $-1<\mathcal{K}<1$,

$$
I_{1}(0)+\left|I_{2}(0)\right|<0 .
$$

In particular

$$
\partial_{\alpha} v_{1}^{p}(0)=I_{1}(0)+I_{2}(0)<I_{1}(0)+\left|I_{2}(0)\right|<0 .
$$

Let us introduce the algorithm we use. We need to compute

$$
\partial_{\alpha} v_{1}^{p}(0)=\int_{0}^{\pi} \ell_{1}+\int_{0}^{\pi} \ell_{2}
$$

where $\ell_{i}$ means the $i$-integral in (5.5). Recall that $\ell_{i}$ is two times differentiable, so, we can use the sharp error bound for the trapezoidal rule. We denote $d x$ the mesh size when we compute the first integral. We approximate the integral of $\ell_{1}$ using the trapezoidal rule between $(0.1, \pi)$. We neglect the integral in the interval $(0,0.1)$, paying with an error denoted by $\left|E_{P V}^{1}\right|=O\left(10^{-3}\right)$. The trapezoidal rule gives us an error $\left|E_{I}^{1}\right| \leqslant \frac{d x^{2}(\pi-0.1)}{12}\left\|\partial_{\alpha}^{2} \ell_{1}\right\|_{L^{\infty}}$. As we know the curve $z$, we can bound $\partial_{\alpha}^{2} \ell_{1}$. We obtain

$$
\left|E_{I}^{1}\right| \leqslant d x^{2} \frac{(\pi-0.1)}{6} 10^{5}
$$


We take $d x=10^{-7}$. Putting all together we obtain

$$
\left|E^{1}\right| \leqslant\left|E_{P V}^{1}\right|+\left|E_{I}^{1}\right|+\leqslant 3 O\left(10^{-3}\right)=O\left(10^{-2}\right) .
$$

Then, we can ensure that

$$
\partial_{\alpha} z_{2}(0) \int_{0}^{\pi} \frac{\partial_{\alpha} z_{1}(\beta) \sin \left(z_{1}(\beta)\right) \sinh \left(z_{2}(\beta)\right)}{\left(\cosh \left(z_{2}(\beta)\right)-\cos \left(z_{1}(\beta)\right)\right)^{2}} d \beta \leqslant-0.7+\left|E^{1}\right|<-0.6 .
$$

We need to control analytically the error in the integral involving $\varpi_{2}^{p}$. This second integral has the error coming form the numerical integration, $E_{I}^{2}$ and a new error coming from the fact that $\varpi_{2}^{p}$ is known with some error. We denote this new error as $E_{w}^{2}$. Let us write $\tilde{d x}$ the mesh size for the second integral. Then, using the smoothness of $\ell_{2}$, we have

$$
\left|E_{I}^{2}\right| \leqslant \frac{\tilde{d x}^{2}}{16}\left\|\varpi_{2}^{p}\right\|_{C^{2}} \leqslant \frac{\tilde{d x}^{2}}{4} \cdot 50 .
$$

We take $\tilde{d x}=10^{-4}$. It remains the error coming from $\varpi_{2}^{p}$. The second vorticity, $\varpi_{2}^{p}$, is given by the integral (5.6). We compute the integral (5.6) using the same mesh size as for $I_{2}, d x$. Thus, the errors are

$$
\left|E_{\varpi}^{2}\right| \leqslant O\left(10^{-3}\right)
$$

Putting all together we have

$$
\left|E^{2}\right| \leqslant\left|E_{I}^{2}\right|+\left|E_{\varpi}^{2}\right| \leqslant O\left(10^{-2}\right),
$$

and we conclude

$$
\left|\frac{\partial_{\alpha} z_{2}(0)}{4 \pi} \int_{0}^{\pi} \frac{\left(\varpi_{2}^{p}(\beta)+\varpi_{2}^{p}(-\beta)\right)\left(-1+\cosh \left(h_{2}\right) \cos (\beta)\right)}{\left(\cosh \left(h_{2}\right)-\cos (\beta)\right)^{2}} d \beta\right| \leqslant 0.1+\left|E^{2}\right|<0.2 .
$$

Now, using (5.23) and (5.24), we obtain $\partial_{\alpha} v_{1}^{p}(0)<0$, and we are done with the periodic case. We proceed with the flat at infinity case. We have to deal with the unboundedness of the domain so we define

$$
z_{1}(\alpha)=\alpha-\sin (\alpha) \exp \left(-\alpha^{2} / 100\right), \quad z_{2}(\alpha)=\frac{\sin (3 \alpha)}{3}-\sin (\alpha)\left(e^{-(\alpha+2)^{2}}+e^{-(\alpha-2)^{2}}\right) \mathbf{1}_{\{|\alpha|<\pi\}} .
$$

Inserting this curve in (5.3) we obtain that for any possible $-1<\mathcal{K}<1$,

$$
I_{1}(0)+\left|I_{2}(0)\right|<0 .
$$

Then, as before,

$$
\partial_{\alpha} v_{1}(0)=I_{1}(0)+I_{2}(0)<I_{1}(0)+\left|I_{2}(0)\right|<0 .
$$

The function $z_{2}$ is Lipschitz, so the same for $\ell_{1}$, where now $\ell_{i}$ are the expressions in (5.3) and the second integral $I_{2}$ is over an unbounded interval. To avoid these problems we compute the numerical approximation of

$$
\int_{0.1}^{\pi-d x} \ell_{1}+\int_{0}^{L_{2}} \ell_{2}
$$


Recall that $\varpi_{2}$ is given by (5.4) and then, due to the definition of $z_{2}$, we can approximate it by an integral over $(0, \pi-\tilde{d x})$. The lack of analyticity of $z_{2}$ and the truncation of $I_{2}(0)$ introduces two new sources of error. We denote them by $E_{z_{2}}^{1}$ and $E_{\mathbb{R}}^{2}$. We take $d x=10^{-7}, \tilde{d x}=10^{-4}$ and $L_{2}=2 \pi$. Using the bounds $z_{1} \leqslant \pi, \partial_{\alpha} z_{1} \leqslant 2$ and $z_{2} \leqslant h_{2}$ we obtain

$$
\left|E_{z_{2}}^{1}\right| \leqslant\left|\int_{\pi-d x}^{\pi} \ell_{1}\right| \leqslant d x \cdot 0.2 \cdot 4 \pi^{2} \leqslant 8 \cdot 10^{-7}
$$

We have

$$
\begin{aligned}
\left|\varpi_{2}(\beta)\right| & \leqslant 4 \pi \frac{\left(h_{2}+\max _{\gamma}\left|z_{2}(\gamma)\right|\right) \max _{\gamma}\left|\partial_{\alpha} z_{2}(\gamma)\right|}{\min _{\gamma}\left(h_{2}+z_{2}(\gamma)\right)^{2}+\left(\beta-z_{1}(\gamma)\right)^{2}} d \gamma \\
& \leqslant \frac{4 \pi \cdot 3 \cdot 2}{\min _{\gamma}\left(h_{2}+z_{2}(\gamma)\right)^{2}+\left(\beta-z_{1}(\gamma)\right)^{2}}=C(\beta),
\end{aligned}
$$

and we get $C(\beta)<C\left(L_{2}\right)$ for $\beta>L_{2}$. Using this inequality we get the desired bound for the second error as follows:

$$
\left|E_{\mathbb{R}}^{2}\right| \leqslant \frac{\left|C\left(L_{2}\right)\right|}{\pi} \int_{L_{2}}^{\infty} \frac{\beta^{2}}{\left(\beta^{2}+\left(\frac{\pi}{2}\right)^{2}\right)^{2}} \leqslant \frac{4 \pi \cdot 3 \cdot 2}{10} \cdot 0.05<4 \cdot 10^{-1} .
$$

The other errors can be bounded as before, obtaining,

$$
\begin{aligned}
& \left|E^{1}\right| \leqslant\left|E_{P V}^{1}\right|+\left|E_{I}^{1}\right|+\left|E_{z_{2}}^{1}\right|=O\left(10^{-2}\right), \\
& \left|E^{2}\right| \leqslant\left|E_{\varpi}^{2}\right|+\left|E_{I}^{2}\right|+\left|E_{\mathbb{R}}^{2}\right|=0.42 .
\end{aligned}
$$

We conclude

$$
\partial_{\alpha} z_{2}(0) \cdot 4 \mathrm{P} . \mathrm{V} . \int_{0}^{\infty} \frac{\partial_{\alpha} z_{1}(\beta) z_{1}(\beta) z_{2}(\beta)}{\left(\left(z_{1}(\beta)\right)^{2}+\left(z_{2}(\beta)\right)^{2}\right)^{2}} d \beta \leqslant-0.7+\left|E^{1}\right|<-0.6,
$$

and

$$
\mid-\frac{1}{2 \pi} \text { P.V. } \int_{0}^{\infty} \frac{\left(\varpi_{2}(\beta)+\varpi_{2}(-\beta)\right) \beta^{2}}{\left(\beta^{2}+h_{2}^{2}\right)^{2}} d \beta|<0.02+| E^{2} \mid<0.5 .
$$

Putting together (5.26) and (5.27) we conclude $\partial_{\alpha} v_{1}(0)<0$.

In order to complete a rigorous enclosure of the integral, we are left with the bounding of the errors coming from the floating point representation and the computer operations and their propagation. In a forthcoming paper (see [18]) we will deal with this matter. By using interval arithmetics we will give a computer assisted proof of this result.

\subsection{Finite depth}

In this section we show the existence of finite time singularities for some curves and physical parameters in an explicit range (see (5.30)). This result is a consequence of Theorem 4 in [14] by means of a continuous dependence on the physical parameters. As a consequence the range of physical parameters plays a role. Indeed, we have 
THEOREM 5.2 Let us suppose that the Rayleigh-Taylor condition is satisfied, i.e. $\rho^{2}-\rho^{1}>0$, and take $0<h_{2}<\frac{\pi}{2}$. There are $f_{0}(x)=f(x, 0) \in H^{3}(\mathbb{R})$, an admissible (see Theorem 3.2) initial datum, such that, for any $|\mathcal{K}|$ small enough, there exists solutions of (1.8) such that $\lim _{t \rightarrow T^{*}}\left\|\partial_{x} f(t)\right\|_{L^{\infty}}=\infty$ for $0<T^{*}<\infty$. For short time $t>T^{*}$ the solution can be continued but it is not a graph.

Proof. The proof is similar to the proof in Theorem 5.1. First, using the result in [14] we obtain a curve, $z(0)$, such that the integrals in $\partial_{\alpha} v_{1}(0)$ coming from $\varpi_{1}$ have a negative contribution. The second step is to take $\mathcal{K}$ small enough, when compared with some quantities depending on the curve $z(0)$, such that the contribution of the terms involving $\varpi_{2}$ is small enough to ensure the singularity. Now, the third step is to prove, using a Cauchy-Kovalevsky theorem, that there exists local in time solutions corresponding to the initial datum $z(0)$. To simplify notation we take $\kappa^{1}\left(\rho^{2}-\rho^{1}\right)=4 \pi$. Then the parameters present in the problem are $h_{2}$ and $\mathcal{K}$.

Obtaining the correct expression. As in [14] and Theorem 5.1 we obtain

$$
\partial_{\alpha} v_{1}(0)=\partial_{t} \partial_{\alpha} z_{1}(0)=I_{1}+I_{2},
$$

where

$$
I_{1}=2 \partial_{\alpha} z_{2}(0) \int_{0}^{\infty} \frac{\partial_{\alpha} z_{1}(\beta) \sinh \left(z_{1}(\beta)\right) \sin \left(z_{2}(\beta)\right)}{\left(\cosh \left(z_{1}(\beta)\right)-\cos \left(z_{2}(\beta)\right)\right)^{2}}+\frac{\partial_{\alpha} z_{1}(\beta) \sinh \left(z_{1}(\beta)\right) \sin \left(z_{2}(\beta)\right)}{\left(\cosh \left(z_{1}(\beta)\right)+\cos \left(z_{2}(\beta)\right)\right)^{2}} d \beta,
$$

and

$$
\begin{aligned}
I_{2}=\frac{\partial_{\alpha} z_{2}(0)}{4 \pi} \int_{\mathbb{R}} \frac{\varpi_{2}(-\beta)\left(-\cosh (\beta) \cos \left(h_{2}\right)+1\right)}{\left(\cosh (\beta)-\cos \left(h_{2}\right)\right)^{2}} d \beta \\
\quad+\frac{\partial_{\alpha} z_{2}(0)}{4 \pi} \int_{\mathbb{R}} \frac{\varpi_{2}(-\beta)\left(-\cosh (\beta) \cos \left(h_{2}\right)-\cos ^{2}\left(h_{2}\right)+\sin ^{2}\left(h_{2}\right)\right)}{\left(\cosh (\beta)+\cos \left(h_{2}\right)\right)^{2}} d \beta .
\end{aligned}
$$

Taking the appropriate curve and $\mathcal{K}$. From Theorem 4 in [14] we know that there are initial curves $w_{0}$ such that $I_{1}=-a^{2}, a=a\left(w_{0}\right)>0$. We take one of this curves and we denote this smooth, fixed curve as $z(0)$. We need to obtain $\mathcal{K}=\mathcal{K}\left(z(0), h_{2}\right)$ such that $\partial_{\alpha} v_{1}(0)=-a^{2}+I_{2}<0$. As in (5.16) we define

$$
d_{1}^{h}[z](\gamma, \beta)=\frac{\cosh ^{2}(\beta / 2)}{\cosh \left(z_{1}(\gamma)-(\gamma-\beta)\right)-\cos \left(z_{2}(\gamma)+h_{2}\right)},
$$

and

$$
d_{2}^{h}[z](\gamma, \beta)=\frac{\cosh ^{2}(\beta / 2)}{\cosh \left(z_{1}(\gamma)-(\gamma-\beta)\right)+\cos \left(z_{2}(\gamma)-h_{2}\right)} .
$$

From the definition of $I_{2}$ it is easy to obtain

$$
\left|I_{2}\right| \leqslant C\left(h_{2}\right) \partial_{\alpha} z_{2}(0)\left\|\varpi_{2}\right\|_{L^{\infty}},
$$


where

$$
C\left(h_{2}\right)=\frac{1}{4 \pi} \int_{\mathbb{R}} \frac{\cosh (\beta) \cos \left(h_{2}\right)+1}{\left(\cosh (\beta)-\cos \left(h_{2}\right)\right)^{2}} d \beta+\frac{1}{4 \pi} \int_{\mathbb{R}} \frac{\cosh (\beta) \cos \left(h_{2}\right)+\cos \left(2 h_{2}\right)}{\left(\cosh (\beta)+\cos \left(h_{2}\right)\right)^{2}} d \beta .
$$

From the definition of $\varpi_{2}$ for curves (which follows from (1.9) in a straightforward way) we obtain

$$
\left\|\varpi_{2}\right\|_{L^{\infty}} \leqslant 8 \mathcal{K}\left\|\partial_{\alpha} z_{2}\right\|_{L^{\infty}}\left(\left\|d_{1}^{h}[z]\right\|_{L^{\infty}}+\left\|d_{2}^{h}[z]\right\|_{L^{\infty}}\right)\left(1+\frac{\mathcal{K}}{\sqrt{2 \pi}}\left\|G_{h_{2}, \mathcal{K}}\right\|_{L^{1}}\right) .
$$

Fixing $0<h_{2}<\pi / 2$ and collecting all the estimates we obtain

$$
\left|I_{2}\right| \leqslant C\left(h_{2}\right) 8 \partial_{\alpha} z_{2}(0) \mathcal{K}\left\|\partial_{\alpha} z_{2}\right\|_{L^{\infty}}\left(\left\|d_{1}^{h}[z]\right\|_{L^{\infty}}+\left\|d_{2}^{h}[z]\right\|_{L^{\infty}}\right)\left(1+\frac{\mathcal{K}}{\sqrt{2 \pi}} \sup _{|\mathcal{K}|<1}\left\|G_{h_{2}, \mathcal{K}}\right\|_{L^{1}}\right) .
$$

Now it is enough to take

$$
\left|\mathcal{K}_{1}\left(z(0), h_{2}\right)\right|<\frac{\left(C\left(h_{2}\right) 8 \partial_{\alpha} z_{2}(0)\left\|\partial_{\alpha} z_{2}\right\|_{L^{\infty}}\right)^{-1} a^{2}}{\left(\left\|d_{1}^{h}[z]\right\|_{L^{\infty}}+\left\|d_{2}^{h}[z]\right\|_{L^{\infty}}\right)\left(1+\frac{1}{\sqrt{2 \pi}} \sup _{|\mathcal{X}|<1} \| G_{h_{2}, \mathcal{K} \|_{L^{1}}}\right)},
$$

to ensure that $\partial_{\alpha} v_{1}(0)<0$ for this curve $z(0)$ and any $|\mathcal{K}|<\left|\mathcal{K}_{1}\left(z(0), h_{2}\right)\right|$.

Showing the forward and backward solvability. We define

$$
d^{-}[z](\gamma, \beta)=\frac{\sinh ^{2}(\beta / 2)}{\cosh \left(z_{1}(\gamma)-z_{1}(\gamma-\beta)\right)-\cos \left(z_{2}(\gamma)-z_{2}(\gamma-\beta)\right)},
$$

and

$$
d^{+}[z](\gamma, \beta)=\frac{\cosh ^{2}(\beta / 2)}{\cosh \left(z_{1}(\gamma)-z_{1}(\gamma-\beta)\right)+\cos \left(z_{2}(\gamma)-z_{2}(\gamma-\beta)\right)} .
$$

Using the equations (5.28), (5.29), (5.31) and (5.32), the proof of this step mimics the proof in Theorem 5.1 and the proof in [14] and so we only sketch it. As before, we consider curves $z$ satisfying the arc-chord condition and such that

$$
\lim _{|\alpha| \rightarrow \infty}|z(\alpha)-(\alpha, 0)|=0 .
$$

We define the complex strip $\mathbb{B}_{r}=\{\zeta+i \xi, \zeta \in \mathbb{R},|\xi|<r\}$, and the spaces (5.10) with norm (5.11) (see [2]). We define the set

$$
\begin{array}{r}
O_{R}=\left\{z \in X_{r} \text { such that }\|z\|_{r}<R,\left\|d^{-}[z]\right\|_{L^{\infty}\left(\mathbb{B}_{r}\right)}<R,\left\|d^{+}[z]\right\|_{L^{\infty}\left(\mathbb{B}_{r}\right)}<R,\right. \\
\left.\left\|d_{1}^{h}[z]\right\|_{L^{\infty}\left(\mathbb{B}_{r}\right)}<R,\left\|d_{2}^{h}[z]\right\|_{L_{\left(\mathbb{B}_{r}\right)}}<R\right\},
\end{array}
$$

where $d_{i}^{h}[z]$ and $d^{ \pm}[z]$ are defined in (5.28), (5.29), (5.31) and (5.32), respectively. As before, we have that, for $z, w \in O_{R}$, complex extension of (2.22), $F: O_{R} \rightarrow X_{r^{\prime}}$ is continuous and the 
following inequalities holds:

$$
\begin{gathered}
\|F[z]\|_{H^{3}\left(\mathbb{B}_{r^{\prime}}\right)} \leqslant \frac{C_{R}}{r-r^{\prime}}\|z\|_{r}, \\
\|F[z]-F[w]\|_{H^{3}\left(\mathbb{B}_{r^{\prime}}\right)} \leqslant \frac{C_{R}}{r-r^{\prime}}\|z-w\|_{H^{3}\left(\mathbb{B}_{r}\right)}, \\
\sup _{\gamma \in \mathbb{B}_{r}, \beta \in \mathbb{R}}|F[z](\gamma)-F[z](\gamma-\beta)| \leqslant C_{R}|\beta| .
\end{gathered}
$$

We consider

$$
z_{n+1}=z(0)+\int_{0}^{t} F\left[z_{n}\right] d s, z_{0}=z(0) .
$$

Using the previous properties of $F$ we obtain that, for $T=T(z(0), R)$ small enough, $z^{n+1} \in O_{R}$, for all $n$. The rest of the proof follows in the same way as in $[25,26]$.

Acknowledgments. The authors are supported by the Grants MTM2011-26696 and SEV-20110087 from Ministerio de Ciencia e Innovación (MICINN). Diego Córdoba was partially supported by StG-203138CDSIF of the ERC. Rafael Granero-Belinchón is grateful to the former Department of Applied Mathematics "Ulisse Dini" of the Pisa University for the hospitality during May-July 2012. We are grateful tInstituto de Ciencias Matemáticas (Madrid) and to the Dipartimento di Ingegneria Aerospaziale (Pisa) for computing facilities.

\section{REFERENCES}

1. Ambrose, D., Well-posedness of two-phase Hele-Shaw flow without surface tension. European J. Appl. Math. 15 (2004), 597-607. Zbl1076.76027 MR2128613

2. Bakan, A. \& Kaijser, S., Hardy spaces for the strip. J. Math. Anal. Appl. 333 (2007), 347-364. Zbl1160.30021 MR2323495

3. BeAR, J., Dynamics of fluids in porous media. Dover Publications, 1988. Zb11191.76002

4. Castro, A., Cordoba, D., Fefferman, C. \& Gancedo, F., Breakdown of smoothness for the Muskat problem. Arch. Ration. Mech. Anal. 208 (2013), 805-909. Zbl06254072 MR3048596

5. Castro, A., Cordoba, D., Fefferman, C., Gancedo, F. \& Lopez-Fernandez, M., RayleighTaylor breakdown for the Muskat problem with applications to water waves. Ann. of Math (2), 175 (2012), 909-948. Zbl1267.76033 MR2993754

6. Cerminara, M. \& FASAnO, A., Modeling the dynamics of a geothermal reservoir fed by gravity driven flow through overstanding saturated rocks. J. Volcanology Geothermal Res. 233-234 (2012), 37-54.

7. Cheng, A., Coutand, D. \& Shkoller, S., Global existence and decay for solutions of the Hele-Shaw flow with injection arXiv preprint arXiv:1208.6213 2012.

8. Constantin, P., Cordoba, D., Gancedo, F. \& Strain, R., On the global existence for the Muskat problem. J. Eur. Math. Soc. 15 (2013), 201-227. Zbl1258.35002 MR2998834

9. Constantin, P. \& M. Pugh. M., Global solutions for small data to the Hele-Shaw problem. Nonlinearity 6 393-415, 1993.

10. Cordoba, A., Córdoba, D. \& Gancedo, F., Interface evolution: the Hele-Shaw and Muskat problems. Ann. of Math (2) $\mathbf{1 7 3}$ (2011), 477-542. Zbl1229. 35204 MR2753607

11. Córdoba, D. \& Gancedo, F., Contour dynamics of incompressible 3-D fluids in a porous medium with different densities. Comm. Math. Phys. 273 (2007), 445-471. Zbl1120.76064 MR2318314 
12. Córdoba, D. \& F. Gancedo, F., A maximum principle for the Muskat problem for fluids with different densities. Comm. Math. Phys. 286 (2009), 681-696. Zb11173. 35637 MR2472040

13. Córdoba, D., GAncedo, F. \& Orive, R., A note on interface dynamics for convection in porous media. Physica D: Nonlinear Phenomena 237 (2008), 1488-1497. Zbl1143.76574

14. Córdoba, D. Granero-Belinchón, R. \& Orive, R., The confined Muskat problem: Differences with the deep water regime. Comm. Math. Sci. 12 (2014), 423-455. MR3144991

15. Escher, J. \& Matioc, B., On the parabolicity of the Muskat problem: Well-posedness, fingering, and stability results. Z. Anal. Anwend. 30 (2011), 193-218.

16. Escher, J., Matioc, A.V. \& Matioc, B., A generalized Rayleigh-Taylor condition for the Muskat problem. Nonlinearity 25 (2011), 73-92. Zbl1243. 35179 MR2864377

17. Escher, J. \& Simonett, G., Classical solutions for Hele-Shaw models with surface tension. $A d v$. Differential Equations 2 (1997), 619-642. Zbl1023. 35527 MR1441859

18. Gómez-Serrano, J. \& Granero-Belinchón, R., On turning waves for the inhomogeneous Muskat problem: a computer-assisted proof, submitted.

19. Hele-Shaw, H., Flow of water by colourbands. Nature $\mathbf{5 8}$ (1897), 520-520, . JFM 29.0646.02

20. Kawarada, H. \& Koshigoe, H., Unsteady flow in porous media with a free surface. Japan J. Indust. Appl. Math. 8 (1991), 41-84. Zb10713.76100 MR1093829

21. KNÜPfer, H. \& N. MAsmoudi, N., Darcy flow on a plate with prescribed contact angle-wellposedness and lubrication approximation. Preprint, arXiv:1204.2278 [math.AP].

22. Majda, A \& Bertozzi, A., Vorticity and incompressible flow. Cambridge Univ. Press, 2002. Zb10983. 76001 MR1867882

23. Musk AT, M., The flow of homogeneous fluids through porous media. McGraw-Hill Publishing Co. (1937). JFM 63.1368.03

24. Nield, D. \& BeJAn, A., Convection in porous media. Springer Verlag, 2006. Zbl1256. 76004

25. Nirenberg, L., An abstract form of the nonlinear Cauchy-Kowalewski theorem. J. Differential Geom 6 (1972), 561-576. Zbl0257.35001 MR0322321

26. NishidA, T., A note on a theorem of Nirenberg. J. Differential Geom 12 (1977), 629-633. Zb10368. 35007 MR0512931

27. Siegel, M., CAFlisCh, R. \& Howison, S., Global existence, singular solutions, and ill-posedness for the Muskat problem. Comm. Pure Appl. Math. 57 (2004), 1374-1411. Zbl1062.35089 MR2070208 